Geometry $\&$ Topology

Volume 7 (2003) 155-184

Published: 14 March 2003

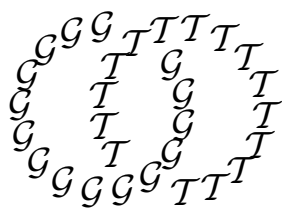

\title{
The smooth Whitehead spectrum of a point at odd regular primes
}

\author{
JoHn ROGNeS \\ Department of Mathematics, University of Oslo \\ N-0316 Oslo, Norway \\ Email: rognes@math.uio.no
}

\begin{abstract}
Let $p$ be an odd regular prime, and assume that the Lichtenbaum-Quillen conjecture holds for $K(\mathbb{Z}[1 / p])$ at $p$. Then the $p$-primary homotopy type of the smooth Whitehead spectrum $W h(*)$ is described. A suspended copy of the cokernel-of-J spectrum splits off, and the torsion homotopy of the remainder equals the torsion homotopy of the fiber of the restricted $S^{1}$-transfer map $t: \Sigma \mathbb{C} P^{\infty} \rightarrow S$. The homotopy groups of $W h(*)$ are determined in a range of degrees, and the cohomology of $W h(*)$ is expressed as an $A$-module in all degrees, up to an extension. These results have geometric topological interpretations, in terms of spaces of concordances or diffeomorphisms of highly connected, high dimensional compact smooth manifolds.
\end{abstract}

AMS Classification numbers Primary: 19D10

Secondary: 19F27, 55P42, 55Q52, 57R50, 57R 80

Keywords: Algebraic $K$-theory, topological cyclic homology, LichtenbaumQuillen conjecture, transfer, $h$-cobordism, concordance, pseudoisotopy

Proposed: Haynes Miller

Seconded: Gunnar Carlsson, Thomas Goodwillie
Received: 30 November 2001 Revised: 7 February 2003

(C) Geometry $\&$ Topology Publications 


\section{Introduction}

In this paper we study the smooth Whitehead spectrum $W h(*)$ of a point at an odd regular prime $p$, under the assumption that the Lichtenbaum-Quillen conjecture for $K(\mathbb{Z}[1 / p])$ holds at $p$. This is a reasonable assumption in view of recent work by Rost and Voevodsky. The results admit geometric topological interpretations in terms of the spaces of concordances (= pseudo-isotopies), $h$-cobordisms and diffeomorphisms of high-dimensional compact smooth manifolds that are as highly connected as their concordance stable range. Examples of such manifolds include discs and spheres.

Here is a summary of the paper.

We begin in section 2 by recalling Waldhausen's algebraic $K$-theory of spaces [49], Quillen's algebraic $K$-theory of rings [33], the Lichtenbaum-Quillen conjecture in the strong formulation of Dwyer and Friedlander [11], and a theorem of Dundas [9] about the relative properties of the cyclotomic trace map to the topological cyclic homology of Bökstedt, Hsiang and Madsen [5].

From section 3 and onwards we assume that $p$ is an odd regular prime and that the Lichtenbaum-Quillen conjecture holds for $K(\mathbb{Z}[1 / p])$ at $p$. In 3.1 and 3.3 we then call on Tate-Poitou duality for étale cohomology [42] to obtain a cofiber sequence

$$
j \vee \Sigma^{-2} k o \rightarrow W h(*) \stackrel{\widetilde{\operatorname{trc}}}{\longrightarrow} \Sigma \mathbb{C} P_{-1}^{\infty} \rightarrow \Sigma j \vee \Sigma^{-1} k o
$$

of implicitly $p$-completed spectra. Here $\mathbb{C} P_{-1}^{\infty}=T h\left(-\gamma^{1}\right)$ is a stunted complex projective spectrum with one cell in each even dimension $\geq-2, j$ is the connective image-of-J spectrum at $p$, and $k o$ is the connective real $K$-theory spectrum. In 3.6 we use this to obtain a splitting

$$
W h(*) \simeq \Sigma c \vee(W h(*) / \Sigma c)
$$

of the suspended cokernel-of-J spectrum $\Sigma c$ off from $W h(*)$, and in 3.8 we obtain a cofiber sequence

$$
\Sigma^{2} k o \rightarrow W h(*) / \Sigma c \stackrel{\tau}{\rightarrow} P_{0} \Sigma \overline{\mathbb{C} P}_{-1}^{\infty} \rightarrow \Sigma^{3} k o,
$$

where $\pi_{*}(\tau)$ identifies the $p$-torsion in the homotopy of $W h(*) / \Sigma c$ with that of $\Sigma \overline{\mathbb{C P}}_{-1}^{\infty}$. The latter spectrum equals the homotopy fiber of the restricted $S^{1}$-transfer map

$$
t: \Sigma \mathbb{C} P^{\infty} \rightarrow S .
$$

Hence the homotopy of $W h(*)$ is as complicated as the (stable) homotopy of infinite complex projective space $\mathbb{C} P^{\infty}$, and the associated transfer map above. 
In section 4 we make a basic homotopical analysis, following Mosher [31] and Knapp [19], to compute $\pi_{*} \Sigma \overline{\mathbb{C} P}_{-1}^{\infty}$ and thus $\pi_{*} W h(*)$ at $p$ in degrees up to $\left|\beta_{2}\right|-2=(2 p+1) q-4$, where $q=2 p-2$ as usual. See 4.7 and 4.9. The first $p$-torsion to appear in $\pi_{m} W h(*)$ is $\mathbb{Z} / p$ for $m=4 p-2$ when $p \geq 5$, and $\mathbb{Z} / 3\left\{\Sigma \beta_{1}\right\}$ for $m=11$ when $p=3$.

In section 5 we make the corresponding mod $p$ cohomological analysis and determine $H^{*}\left(W h(*) ; \mathbb{F}_{p}\right)$ as a module over the Steenrod algebra is all degrees, up to an extension. See 5.4 and 5.5. The extension is trivial for $p=3$, and nontrivial for $p \geq 5$. Taken together, this homotopical and cohomological information gives a detailed picture of the homotopy type $W h(*)$.

In section 6 we recall the relation between the Whitehead spectrum $W h(*)$, the concordance space $C(M)$ and the diffeomorphism group $D I F F(M)$ of suitably highly connected and high dimensional compact smooth manifolds $M$. As a sample application we show in 6.3 that for $p \geq 5$ and $M$ a compact smooth $k$-connected $n$-manifold with $k \geq 4 p-2$ and $n \geq 12 p-5$, the first $p$-torsion in the homotopy of the smooth concordance space $C(M)$ is $\pi_{4 p-4} C(M)_{(p)} \cong$ $\mathbb{Z} / p$. Specializing to $M=D^{n}$ we conclude in 6.4 that $\pi_{4 p-4} D I F F\left(D^{n+1}\right)$ or $\pi_{4 p-4} D I F F\left(D^{n}\right)$ contains an element of order exactly $p$. Comparable results hold for $p=3$.

A 2-primary analog of this study was presented in [38]. Related results on the homotopy fiber of the linearization map $L: A(*) \rightarrow K(\mathbb{Z})$ were given in [18].

\section{Algebraic $K$-theory and topological cyclic homo- $\log y$}

\section{Algebraic $K$-theory of spaces}

Let $A(X)$ be Waldhausen's algebraic $K$-theory spectrum [49, section 2.1] of a space $X$. There is a natural cofiber sequence [49, section 3.3], [50]

$$
\Sigma^{\infty}\left(X_{+}\right) \stackrel{\eta_{X}}{\longrightarrow} A(X) \stackrel{\pi}{\longrightarrow} W h(X),
$$

where $W h(X)=W h^{D I F F}(X)$ is the smooth Whitehead spectrum of $X$, and a natural trace map [47] $\operatorname{tr}_{X}: A(X) \rightarrow \Sigma^{\infty}\left(X_{+}\right)$which splits the above cofiber sequence up to homotopy. Let $\iota: W h(X) \rightarrow A(X)$ be the corresponding homotopy section to $\pi$. When $X=*$ is a point, $\Sigma^{\infty}\left(*_{+}\right)=S$ is the sphere spectrum, and the splitting simplifies to $A(*) \simeq S \vee W h(*)$. 


\section{Topological cyclic homology of spaces}

Let $p$ be a prime and let $T C(X ; p)$ be Bökstedt, Hsiang and Madsen's topological cyclic homology $[5,5.12(\mathrm{i})]$ of the space $X$. There is a natural cofiber sequence $[5,5.17]$

$$
\operatorname{hofib}\left(\operatorname{trf}_{S^{1}}\right) \stackrel{\iota}{\rightarrow} T C(X ; p) \stackrel{\beta_{X}}{\longrightarrow} \Sigma^{\infty}\left(\Lambda X_{+}\right)
$$

after $p$-adic completion, where $\Lambda X$ is the free loop space of $X$ and

$$
\operatorname{trf}_{S^{1}}: \Sigma^{\infty}\left(\Sigma\left(E S^{1} \times_{S^{1}} \Lambda X\right)_{+}\right) \rightarrow \Sigma^{\infty}\left(\Lambda X_{+}\right)
$$

is the dimension-shifting $S^{1}$-transfer map for the canonical $S^{1}$-bundle $E S^{1} \times$ $\Lambda X \rightarrow E S^{1} \times_{S^{1}} \Lambda X$; see e.g. [23, section 2]. When $X=*$ the $S^{1}$-transfer map simplifies to $\operatorname{trf}_{S^{1}}: \Sigma^{\infty} \Sigma \mathbb{C} P_{+}^{\infty} \rightarrow S$. Its homotopy fiber is $\Sigma \mathbb{C} P_{-1}^{\infty}[23$, section 3], where the stunted complex projective spectrum $\mathbb{C} P_{-1}^{\infty}=T h\left(-\gamma^{1} \downarrow\right.$ $\left.\mathbb{C} P^{\infty}\right)$ is defined as the Thom spectrum of minus the tautological line bundle over $\mathbb{C} P^{\infty}$. The map $\iota$ identifies $\Sigma \mathbb{C} P_{-1}^{\infty}$ with the homotopy fiber of $\beta_{*}: T C(* ; p) \rightarrow S$, after $p$-adic completion.

We can think of $\mathbb{C} P_{-1}^{\infty}$ as a CW spectrum, with $2 k$-skeleton $\mathbb{C} P_{-1}^{k}=T h\left(-\gamma^{1} \downarrow\right.$ $\left.\mathbb{C} P^{k+1}\right)$. By James periodicity $\Sigma^{2 n} \mathbb{C} P_{-1}^{k} \simeq \mathbb{C} P_{n-1}^{n+k}=\mathbb{C} P^{n+k} / \mathbb{C} P^{n-2}$ whenever $n$ is a multiple of a suitable natural number that depends on $k$. From this it follows that integrally $H_{*}\left(\mathbb{C} P_{-1}^{\infty}\right) \cong \mathbb{Z}\left\{b_{k} \mid k \geq-1\right\}$ and $H^{*}\left(\mathbb{C} P_{-1}^{\infty}\right) \cong \mathbb{Z}\left\{y^{k} \mid\right.$ $k \geq-1\}$ with $y^{k}$ dual to $b_{k}$, both in degree $2 k$. In mod $p$ cohomology the Steenrod operations act by $P^{i}\left(y^{k}\right)=\left(\begin{array}{c}k \\ i\end{array}\right) y^{k+(p-1) i}$ and $\beta\left(y^{k}\right)=0$. In particular $P^{i}\left(y^{-1}\right)=(-1)^{i} y^{-1+(p-1) i} \neq 0$ for all $i \geq 0$.

\section{The cyclotomic trace map for spaces}

Let $\operatorname{trc}_{X}: A(X) \rightarrow T C(X ; p)$ be the natural cyclotomic trace map of Bökstedt, Hsiang and Madsen [5, 5.12(ii)]. It lifts the Waldhausen trace map, in the sense that $\operatorname{tr}_{X} \simeq$ ev $\circ \beta_{X} \circ \operatorname{trc}_{X}$, where ev: $\Sigma^{\infty}\left(\Lambda X_{+}\right) \rightarrow \Sigma^{\infty}\left(X_{+}\right)$evaluates a free loop at a base point. Hence there is a map of (split) cofiber sequences of spectra:

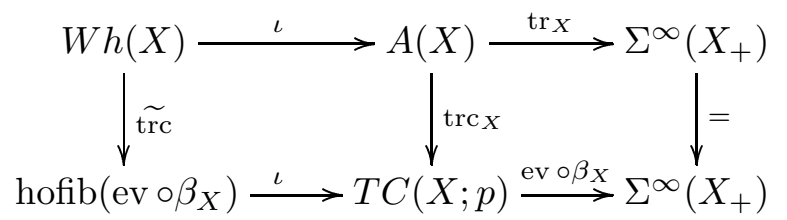

after $p$-adic completion. When $X=*$ the left hand square simplifies as follows: 
Theorem 2.1 (Waldhausen, Bökstedt-Hsiang-Madsen) There is a homotopy Cartesian square

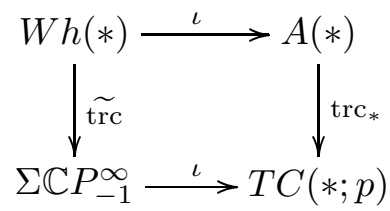

after $p$-adic completion. Hence there is a $p$-complete equivalence hofib $(\widetilde{\operatorname{tr} c}) \simeq$ $\operatorname{hofib}\left(\operatorname{trc}_{*}\right)$.

\section{Algebraic $K$-theory of rings}

Let $K(R)$ be Quillen's algebraic $K$-theory spectrum of a ring $R$ [33, section 2]. When $R$ is commutative, Noetherian and $1 / p \in R$ the étale $K$-theory spectrum $K^{\text {ét }}(R)$ of Dwyer and Friedlander [11, section 4] is defined, and comes equipped with a natural comparison map $\phi: K(R) \rightarrow K^{\text {ét }}(R)$. By construction $K^{\text {ét }}(R)$ is a $p$-adically complete $K$-local spectrum [8]. Let $R$ be the ring of $p$-integers in a local or a global field of characteristic $\neq p$. The Lichtenbaum-Quillen conjecture [20], [21], [35] for $K(R)$ at $p$, in the strong form due to Dwyer and Friedlander, then asserts:

Conjecture 2.2 (Lichtenbaum-Quillen) The comparison map $\phi$ induces a homotopy equivalence

$$
P_{1} \phi_{p}^{\wedge}: P_{1} K(R)_{p}^{\wedge} \rightarrow P_{1} K^{\mathrm{ét}}(R)
$$

of 0 -connected covers after $p$-adic completion.

Here $P_{n} E$ denotes the $(n-1)$-connected cover of any spectrum $E$. In the cases of concern to us the $p$-completed map $\phi_{p}^{\wedge}$ will also induce an isomorphism in degree 0 , so the covers $P_{1}$ above can be replaced by $P_{0}$.

The conjecture above has been proven for $p=2$ by Rognes and Weibel [39, 0.6], based on Voevodsky's proof [44], [45] of the Milnor conjecture. The oddprimary version of this conjecture would follow [41] from results on the BlochKato conjecture [4] announced as "in preparation" by Rost and Voevodsky, but have not yet formally appeared. 


\section{Topological cyclic homology of rings}

Let $T C(R ; p)$ be Bökstedt, Hsiang and Madsen's topological cyclic homology of a (general) ring $R$. There is a natural cyclotomic trace map $\operatorname{trc}_{R}: K(R) \rightarrow$ $T C(R ; p)$. When $X$ is a based connected space with fundamental group $\pi=$ $\pi_{1}(X)$, and $R=\mathbb{Z}[\pi]$ is the group ring, there are natural linearization maps $L: A(X) \rightarrow K(R)[46$, section 2$]$ and $L: T C(X ; p) \rightarrow T C(R ; p)$ which commute with the cyclotomic trace maps. Moreover, by Dundas [9] the square

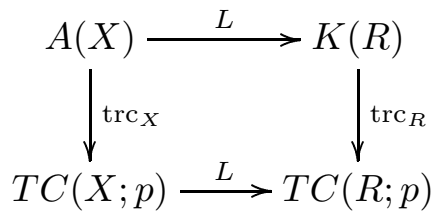

is homotopy Cartesian after $p$-adic completion. In the special case when $X=*$ and $R=\mathbb{Z}$ this simplifies to:

Theorem 2.3 (Dundas) There is a homotopy Cartesian square

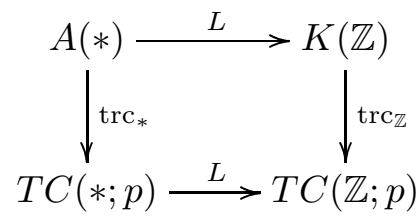

after $p$-adic completion. Hence there is a $p$-complete equivalence hofib $\left(\operatorname{trc}_{*}\right) \simeq$ hofib $\left(\operatorname{trc}_{\mathbb{Z}}\right)$.

\section{The cyclotomic trace map for rings}

When $k$ is a perfect field of characteristic $p>0, W(k)$ its ring of Witt vectors, and $R$ is an algebra of finite rank over $W(k)$, then by Hesselholt and Madsen $[15$, Thm. D] there is a cofiber sequence of spectra

$$
K(R) \stackrel{\operatorname{trc}_{R}}{\longrightarrow} T C(R ; p) \rightarrow \Sigma^{-1} H W(R)_{F}
$$

after $p$-adic completion. Here $W(R)_{F}$ equals the coinvariants of the Frobenius action on the Witt ring of $R$, and $\Sigma^{-1} H W(R)_{F}$ is the associated desuspended Eilenberg-Mac Lane spectrum. The Witt ring of $k=\mathbb{F}_{p}$ is the ring $W\left(\mathbb{F}_{p}\right)=\mathbb{Z}_{p}$ of $p$-adic integers, so the above applies to $R=\mathbb{Z}_{p}[\pi]$ for finite groups $\pi$. In particular, when $X=*$ and $\pi=1$ there is a cofiber sequence

$$
K\left(\mathbb{Z}_{p}\right) \stackrel{\operatorname{trc}_{\mathbb{Z}_{p}}}{\longrightarrow} T C\left(\mathbb{Z}_{p} ; p\right) \rightarrow \Sigma^{-1} H \mathbb{Z}_{p}
$$

after $p$-adic completion. This uses that $W\left(\mathbb{Z}_{p}\right)_{F} \cong \mathbb{Z}_{p}$. 


\section{The completion map}

Let $\kappa: \mathbb{Z} \rightarrow \mathbb{Z}_{p}$ and $\kappa^{\prime}: \mathbb{Z}[1 / p] \rightarrow \mathbb{Q}_{p}$ be the $p$-completion homomorphisms, where $\mathbb{Q}_{p}$ is the field of $p$-adic numbers. By naturality of $\operatorname{trc}_{R}$ with respect to $\kappa$ there is a commutative square

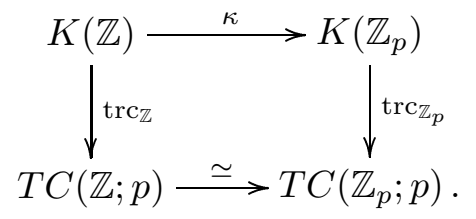

The lower map is a $p$-adic equivalence, since topological cyclic homology is insensitive to $p$-adic completion, cf. [15, section 6]. Hence there is a cofiber sequence of homotopy fibers

$$
\operatorname{hofib}(\kappa) \rightarrow \operatorname{hofib}\left(\operatorname{trc}_{\mathbb{Z}}\right) \rightarrow \Sigma^{-2} H \mathbb{Z}_{p} .
$$

By the localization sequences in $K$-theory [33, section 5] there is a homotopy Cartesian square

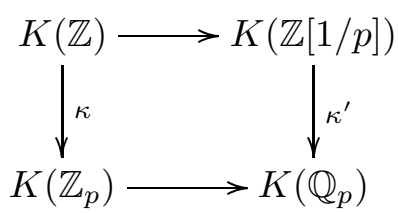

so $\operatorname{hofib}(\kappa) \simeq \operatorname{hofib}\left(\kappa^{\prime}\right)$.

\section{Topological $K$-theory and related spectra}

Let $k o$ and $k u$ be the connective real and complex topological $K$-theory spectra, respectively. There is a complexification map $c: k o \rightarrow k u$, and a cofiber sequence

$$
\Sigma k o \stackrel{\eta}{\rightarrow} k o \stackrel{c}{\rightarrow} k u \stackrel{r \beta^{-1}}{\longrightarrow} \Sigma^{2} k o
$$

related to real Bott periodicity, cf. [26, V.5.15]. Here $\eta$ is multiplication by the stable Hopf map $\eta: S^{1} \rightarrow S^{0}$, which is null-homotopic at odd primes, $\beta: \Sigma^{2} k u \rightarrow k u$ covers the Bott equivalence, and $r: k u \rightarrow k o$ is realification.

Suppose $p$ is odd, and let $q=2 p-2$. There are splittings $k u_{(p)} \simeq \bigvee_{i=0}^{p-2} \Sigma^{2 i} \ell$ and

$$
k o_{(p)} \simeq \bigvee_{i=0}^{(p-3) / 2} \Sigma^{4 i} \ell,
$$


where $\ell$ is the connective $p$-local Adams summand of $k u$ [1]. There is a cofiber sequence $\Sigma^{q} \ell \rightarrow \ell \rightarrow H \mathbb{Z}_{(p)}$ that identifies $\Sigma^{q} \ell$ with $P_{q} \ell$. Let $r$ be a topological generator of the $p$-adic units $\mathbb{Z}_{p}^{*}$, and let $\psi^{r}$ be the Adams operation. The $p$ local image-of-J spectrum $j$ is defined [26, V.5.16] by the cofiber sequence

$$
j \rightarrow \ell \stackrel{\psi^{r}-1}{\longrightarrow} \Sigma^{q} \ell .
$$

We now briefly write $S$ for the $p$-local sphere spectrum. There is a unit map $e: S \rightarrow j$ representing (minus) the Adams $e$-invariant on homotopy [36], and the $p$-local cokernel-of-J spectrum $c$ is defined by the cofiber sequence

$$
c \stackrel{f}{\rightarrow} S \stackrel{e}{\rightarrow} j .
$$

Here $e$ induces a split surjection on homotopy, so $\pi_{*}(f)$ is split injective. The map $e$ identifies $j$ with the connective cover $P_{0} L_{K} S$ of the $K$-localization of $S$, localized at $p[8,4.3]$.

Lemma 2.6 Suppose that $n \leq 2 q$. If $n \neq q+1$ there are no essential spectrum maps $H \mathbb{Z}_{(p)} \rightarrow \Sigma^{n} \ell$. If $n=q+1$ the group of spectrum maps $H \mathbb{Z}_{(p)} \rightarrow \Sigma^{q+1} \ell$ is $\mathbb{Z}_{(p)}$, generated by the connecting map $\partial$ of the cofiber sequence $\Sigma^{q} \ell \rightarrow \ell \rightarrow H \mathbb{Z}_{(p)}$.

Lemma 2.7 There are no essential spectrum maps $\Sigma^{n} \ell \rightarrow j$ for $n \geq 0$ even. Hence there are no essential spectrum maps $\Sigma k_{(p)} \rightarrow \Sigma j$.

The proofs are easy, using [29] for 2.6 , and [24, Cor. C] or [30, 2.4] for 2.7 .

\section{Splittings at odd regular primes}

\section{The completion map in étale $K$-theory}

When $R=\mathbb{Z}[1 / p]$ and $p$ is an odd regular prime there is a homotopy equivalence $P_{0} K^{\text {ét }}(\mathbb{Z}[1 / p]) \simeq j \vee \Sigma k o$ after $p$-adic completion $[12,2.3]$. Taking into account that $\phi$ is an equivalence in degree 0 and that $K(\mathbb{Z}[1 / p])$ has finite type [34], the Lichtenbaum-Quillen conjecture for $\mathbb{Z}[1 / p]$ at $p$ amounts to the assertion that $K(\mathbb{Z}[1 / p]) \simeq j \vee \Sigma k o$ after $p$-localization. By the localization sequence in $K$-theory, this is equivalent to the assertion that $K(\mathbb{Z}) \simeq j \vee \Sigma^{5} k o$, after $p$-localization.

Hereafter we (often implicitly) complete all spectra at $p$. 
When $R=\mathbb{Q}_{p}$ and $p$ is an odd prime there is a $p$-adic equivalence $P_{0} K^{\text {ét }}\left(\mathbb{Q}_{p}\right) \simeq$ $j \vee \Sigma j \vee \Sigma k u$. The Lichtenbaum-Quillen conjecture for $\mathbb{Q}_{p}$ at $p$ asserts that $K\left(\mathbb{Q}_{p}\right) \simeq j \vee \Sigma j \vee \Sigma k u[13,13.3]$, which again is equivalent to the assertion that $K\left(\mathbb{Z}_{p}\right) \simeq j \vee \Sigma j \vee \Sigma^{3} k u$, after $p$-adic completion. This is now a theorem, following from the calculation by Bökstedt and Madsen of $T C(\mathbb{Z} ; p)$ [6, 9.17], [7].

Proposition 3.1 Let $p$ be an odd regular prime. There are $p$-adic equivalences $P_{0} K^{\text {ét }}(\mathbb{Z}[1 / p]) \simeq j \vee \Sigma k o$ and $P_{0} K^{\text {ét }}\left(\mathbb{Q}_{p}\right) \simeq j \vee \Sigma j \vee \Sigma k u$ such that

$$
\kappa^{\prime}: P_{0} K^{\text {ét }}(\mathbb{Z}[1 / p]) \rightarrow P_{0} K^{\text {ét }}\left(\mathbb{Q}_{p}\right)
$$

is homotopic to the wedge sum of the identity $i d: j \rightarrow j$, the zero map $* \rightarrow \Sigma j$, and the suspended complexification map $\Sigma c: \Sigma k o \rightarrow \Sigma k u$. Thus hofib $\left(\kappa^{\prime}\right) \simeq$ $j \vee \Sigma^{2} k o$.

Proof Taking the topological generator $r$ to be a prime power, there is a reduction map red: $P_{0} K^{\text {ét }}\left(\mathbb{Q}_{p}\right) \rightarrow K\left(\mathbb{F}_{r}\right) \simeq j$ after $p$-adic completion [13, section 13], such that the composite map

$$
S \stackrel{\eta}{\rightarrow} K(\mathbb{Z}[1 / p]) \stackrel{\phi}{\longrightarrow} P_{0} K^{\text {ét }}(\mathbb{Z}[1 / p]) \stackrel{\kappa^{\prime}}{\longrightarrow} P_{0} K^{\text {ét }}\left(\mathbb{Q}_{p}\right) \stackrel{\text { red }}{\longrightarrow} j
$$

is homotopic to $e$. Since $K^{\text {ét }}(\mathbb{Z}[1 / p])$ is $K$-local, $\phi \eta$ also factors through $e$. These maps split off a common copy of $j$ from $P_{0} K^{\text {ét }}(\mathbb{Z}[1 / p])$ and $P_{0} K^{\text {ét }}\left(\mathbb{Q}_{p}\right)$. There are no essential spectrum maps $\Sigma k o \rightarrow \Sigma j$ by 2.7 , so after $p$-adic completion $\kappa^{\prime}$ is homotopic to a wedge sum of maps $i d: j \rightarrow j, * \rightarrow \Sigma j$ and a map $\kappa^{\prime \prime}: \Sigma k o \rightarrow \Sigma k u$. Any such $\kappa^{\prime \prime}$ lifts over $\Sigma c: \Sigma k o \rightarrow \Sigma k u$, so it suffices to show that $\pi_{2 i-1}\left(\kappa^{\prime \prime}\right)$ is a $p$-adic isomorphism for all odd $i \geq 1$.

Equivalently we must show that $\kappa^{\prime}$ induces an isomorphism on homotopy modulo torsion subgroups in degree $2 i-1$ for all odd $i>1$, or that

$$
K_{2 i-1}^{\text {ét }}\left(\kappa^{\prime} ; \mathbb{Q}_{p} / \mathbb{Z}_{p}\right): K_{2 i-1}^{\text {ét }}\left(\mathbb{Z}[1 / p] ; \mathbb{Q}_{p} / \mathbb{Z}_{p}\right) \rightarrow K_{2 i-1}^{\text {ét }}\left(\mathbb{Q}_{p} ; \mathbb{Q}_{p} / \mathbb{Z}_{p}\right)
$$

is injective. This equals the completion map

$$
\kappa^{\prime}: H_{\text {ét }}^{1}\left(\mathbb{Z}[1 / p] ; \mathbb{Q}_{p} / \mathbb{Z}_{p}(i)\right) \rightarrow H_{\text {ét }}^{1}\left(\mathbb{Q}_{p} ; \mathbb{Q}_{p} / \mathbb{Z}_{p}(i)\right)
$$

in étale cohomology, by the collapsing spectral sequence in [, 5.1]. By the 9-term exact sequence expressing Tate-Poitou duality [42, 3.1], [28, I.4.10], its kernel is a quotient of $A^{\#}=H_{\text {êt }}^{2}\left(\mathbb{Z}[1 / p] ; \mathbb{Z}_{p}(1-i)\right)^{\#}$, where $A^{\#}=\operatorname{Hom}(A, \mathbb{Q} / \mathbb{Z})$ denotes the Pontryagin dual of an abelian group $A$. But $A=H_{\text {êt }}^{2}\left(\mathbb{Z}[1 / p] ; \mathbb{Z}_{p}(1-i)\right)$ is an abelian pro- $p$-group, with $A / p \cong H_{\text {ét }}^{2}(\mathbb{Z}[1 / p] ; \mathbb{Z} / p(1-i))$ contained as a direct summand in $B=H_{\text {êt }}^{2}\left(\mathbb{Z}\left[1 / p, \zeta_{p}\right] ; \mathbb{Z} / p\right)$, which is independent of $i$. Here 
$R=\mathbb{Z}\left[1 / p, \zeta_{p}\right]$ is the ring of $p$-integers in the $p$-th cyclotomic field $\mathbb{Q}\left(\zeta_{p}\right)$. Kummer theory gives a short exact sequence

$$
0 \rightarrow \operatorname{Pic}(R) / p \rightarrow B \rightarrow\{p\} \operatorname{Br}(R) \rightarrow 0
$$

where $\operatorname{Pic}(R)$ and $\operatorname{Br}(R)$ are the Picard and Brauer groups of $R$, respectively. (See [28, section IV] and [16].) Here $\operatorname{Pic}(R) / p=0$ because $p$ is a regular prime, and $\{p\} \operatorname{Br}(R)=\operatorname{ker}(p: \operatorname{Br}(R) \rightarrow \operatorname{Br}(R))=0$ because $p$ is odd and $(p)$ does not split in $R$ [27, p. 109], so $B=0$. Thus $A / p=0$ and it follows that $A=0$, since $A$ is an abelian pro- $p$-group.

\section{The fiber of the cyclotomic trace map}

Hereafter we make the following standing assumption.

Hypothesis 3.2 (a) $p$ is an odd regular prime, and

(b) the Lichtenbaum-Quillen conjecture 2.2 holds for $K(\mathbb{Z}[1 / p])$ at $p$.

Proposition 3.3 There is a homotopy equivalence hofib $\left(\operatorname{trc}_{\mathbb{Z}}\right) \simeq j \vee \Sigma^{-2} k o$ after $p$-adic completion.

Proof By assumption $\kappa^{\prime}: K(\mathbb{Z}[1 / p]) \rightarrow K\left(\mathbb{Q}_{p}\right)$ agrees with

$$
\kappa^{\prime}: P_{0} K^{\text {ét }}(\mathbb{Z}[1 / p]) \rightarrow P_{0} K^{\text {ét }}\left(\mathbb{Q}_{p}\right)
$$

after $p$-adic completion, so we have a cofiber sequence

$$
j \vee \Sigma^{2} k o \rightarrow \operatorname{hofib}\left(\operatorname{trc}_{\mathbb{Z}}\right) \rightarrow \Sigma^{-2} H \mathbb{Z}_{p} .
$$

The connecting map $\Sigma^{-2} H \mathbb{Z}_{p} \rightarrow \Sigma j \vee \Sigma^{3} k o$ is homotopic to a wedge sum of maps $\Sigma^{-2} H \mathbb{Z}_{p} \rightarrow \Sigma j$ and $\Sigma^{-2} H \mathbb{Z}_{p} \rightarrow \Sigma^{4 i-1} \ell$ for $1 \leq i \leq(p-1) / 2$. All such maps are null-homotopic by 2.6 , with the exception of the map $\partial^{\prime}: \Sigma^{-2} H \mathbb{Z}_{p} \rightarrow$ $\Sigma^{2 p-3} \ell$ corresponding to $i=(p-1) / 2$.

We claim that multiplication by $v_{1}$ acts nontrivially from degree -2 to degree $2 p-4$ in $\pi_{*}\left(\right.$ hofib $\left.\left(\operatorname{trc}_{\mathbb{Z}}\right) ; \mathbb{Z} / p\right)$, from which it follows that $\partial^{\prime}$ is a $p$-adic unit times the connecting map $\partial$ in the cofiber sequence $\Sigma^{q-2} \ell \rightarrow \Sigma^{-2} \ell \rightarrow \Sigma^{-2} H \mathbb{Z}_{p}$. This implies that

$$
\operatorname{hofib}\left(\operatorname{trc}_{\mathbb{Z}}\right) \simeq j \vee \Sigma^{-2} \ell \vee \bigvee_{i=1}^{(p-3) / 2} \Sigma^{4 i-2} \ell \simeq j \vee \Sigma^{-2} k o .
$$

To prove the claim, consider the homotopy Cartesian squares in 2.1 and 2.3. In the Atiyah-Hirzebruch spectral sequence

$$
E_{s, t}^{2}=H_{s}\left(\mathbb{C} P_{-1}^{\infty} ; \pi_{t}(S ; \mathbb{Z} / p)\right) \Longrightarrow \pi_{s+t}\left(\mathbb{C} P_{-1}^{\infty} ; \mathbb{Z} / p\right)
$$


there is a first differential $d^{2 p-2}\left(b_{p-2}\right)=\alpha_{1} b_{-1}$, so we find $\pi_{-2}\left(\mathbb{C} P_{-1}^{\infty} ; \mathbb{Z} / p\right) \cong$ $\mathbb{Z} / p\left\{b_{-1}\right\}$ and $\pi_{2 p-4}\left(\mathbb{C} P_{-1}^{\infty} ; \mathbb{Z} / p\right) \cong \mathbb{Z} / p\left\{v_{1} b_{-1}\right\}$. Hence multiplication by $v_{1}$ acts nontrivially from

$$
\pi_{-1}(T C(* ; p) ; \mathbb{Z} / p) \cong \mathbb{Z} / p\left\{\Sigma b_{-1}\right\}
$$

to

$$
\pi_{2 p-3}(T C(* ; p) ; \mathbb{Z} / p) \cong \mathbb{Z} / p\left\{\alpha_{1}, \Sigma v_{1} b_{-1}\right\},
$$

also modulo the image from the unit map $\eta: S \rightarrow T C(* ; p)$.

The map $L: S \rightarrow H \mathbb{Z}$ is $(2 p-3)$-connected, hence so is $L: T C(* ; p) \rightarrow$ $T C(\mathbb{Z} ; p)$ by $[6,10.9]$ and $[9]$. Here $\pi_{2 p-3}(T C(\mathbb{Z} ; p) ; \mathbb{Z} / p) \cong \mathbb{Z} / p\left\{\alpha_{1}\right\} \oplus \mathbb{Z} / p$ since $P_{0} T C(\mathbb{Z} ; p) \simeq K\left(\mathbb{Z}_{p}\right) \simeq j \vee \Sigma j \vee \Sigma^{3} k u$. So the surjection $\pi_{2 p-3}(L ; \mathbb{Z} / p)$ is in fact a bijection, and multiplication by $v_{1}$ acts nontrivially from $\pi_{-1}(T C(\mathbb{Z} ; p) ; \mathbb{Z} / p)$ to $\pi_{2 p-3}(T C(\mathbb{Z} ; p) ; \mathbb{Z} / p)$, also modulo the image from the unit map $\eta: S \rightarrow$ $T C(\mathbb{Z} ; p)$.

By the assumed $p$-adic equivalence $K(\mathbb{Z}) \simeq j \vee \Sigma^{5} k o$, this image equals the image from the cyclotomic trace map $\operatorname{trc}_{\mathbb{Z}}: K(\mathbb{Z}) \rightarrow T C(\mathbb{Z} ; p)$. Hence we can pass to cofibers, and conclude that multiplication by $v_{1}$ acts nontrivially from $\pi_{-2}\left(\right.$ hofib $\left.\left(\operatorname{trc}_{\mathbb{Z}}\right) ; \mathbb{Z} / p\right)$ to $\pi_{2 p-4}\left(\operatorname{hofib}\left(\operatorname{trc}_{\mathbb{Z}}\right) ; \mathbb{Z} / p\right)$, as claimed.

We let $d$ be the homotopy cofiber map of $\widetilde{\text { trc }}$. Combining 2.1, 2.3 and 3.3 we have:

Corollary 3.4 There is a diagram of horizontal cofiber sequences:

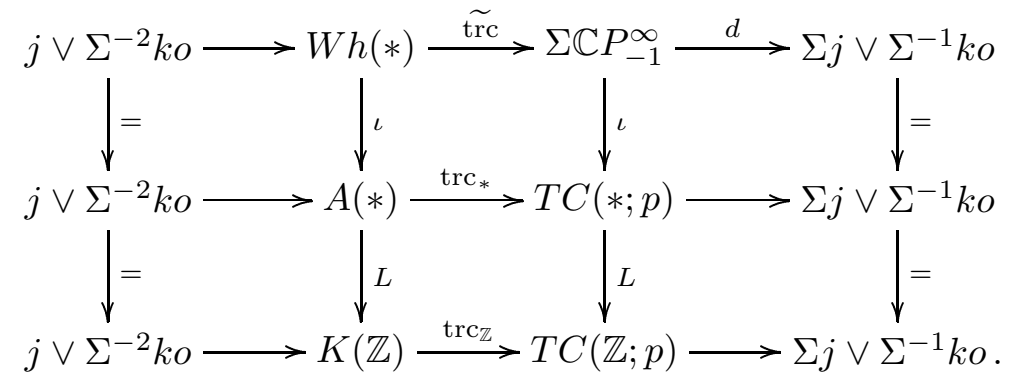

\section{The restricted $S^{1}$-transfer map}

There is a stable splitting $\operatorname{in}_{1} \vee \operatorname{in}_{2}: S^{1} \vee \Sigma \mathbb{C} P^{\infty} \simeq \Sigma \mathbb{C} P_{+}^{\infty}$. Let the restricted $S^{1}$-transfer map $t=\operatorname{trf}_{S^{1}} \circ \mathrm{in}_{2}: \Sigma \mathbb{C} P^{\infty} \rightarrow S$ be the restriction of $\operatorname{trf}_{S^{1}}$ to the second summand [32, section 2]. The restriction to the first summand is the stable Hopf map $\eta=\operatorname{trf}_{S^{1}} \circ \mathrm{in}_{1}: S^{1} \rightarrow S^{0}$, which is null-homotopic at 
odd primes. Hence the inclusion in in $_{1}$ lifts to a map $\Sigma b_{0}: S^{1} \rightarrow$ hofib $\left(\operatorname{trf}_{S^{1}}\right)=$ $\Sigma \mathbb{C} P_{-1}^{\infty}$, with Hurewicz image $\Sigma b_{0} \in H_{1}\left(\Sigma \mathbb{C} P_{-1}^{\infty}\right)$.

Dually the projection $\mathrm{pr}_{1}: \Sigma \mathbb{C} P_{+}^{\infty} \rightarrow S^{1}$ yields a map $\Sigma y^{0}: \Sigma \mathbb{C} P_{-1}^{\infty} \rightarrow S^{1}$ with dual Hurewicz image $\Sigma y^{0} \in H^{1}\left(\Sigma \mathbb{C} P_{-1}^{\infty}\right)$. We obtain a diagram of horizontal and split vertical cofiber sequences:

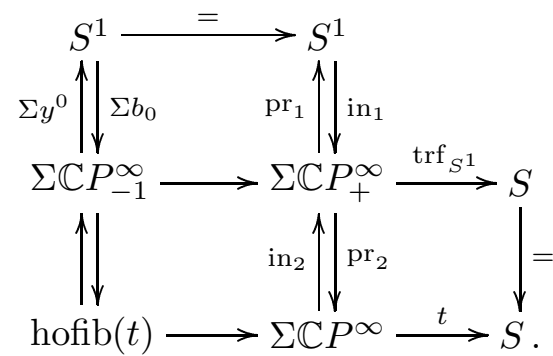

Writing $\overline{\mathbb{C P}}_{-1}^{\infty}$ for the homotopy cofiber of $b_{0}: S \rightarrow \mathbb{C} P_{-1}^{\infty}$, we have hofib $(t) \simeq$ $\Sigma \overline{\mathbb{C} P}_{-1}^{\infty}$. Then $H_{*}\left(\Sigma \overline{\mathbb{C} P}_{-1}^{\infty}\right)=\mathbb{Z}\left\{\Sigma b_{k} \mid k \geq-1, k \neq 0\right\}$ and $H^{*}\left(\Sigma \overline{\mathbb{C P}}_{-1}^{\infty}\right) \cong$ $\mathbb{Z}\left\{\Sigma y^{k} \mid k \geq-1, k \neq 0\right\}$.

It has been shown by Knapp [19] that $\pi_{*}(t): \pi_{*}\left(\Sigma \mathbb{C} P^{\infty}\right) \rightarrow \pi_{*}(S)$ is surjective for $0<*<\left|\beta_{p+1}\right|=p(p+2) q-2$, so the homotopy of $\Sigma \overline{\mathbb{C} P}_{-1}^{\infty}$ is as well understood in this range as that of $\Sigma \mathbb{C} P^{\infty}$.

\section{The suspended cokernel-of-J spectrum}

We can split off the suspension of the cofiber sequence (2.5) defining the cokernelof-J from the top cofiber sequence in 3.4.

Proposition 3.6 There is a diagram of horizontal and split vertical cofiber sequences:

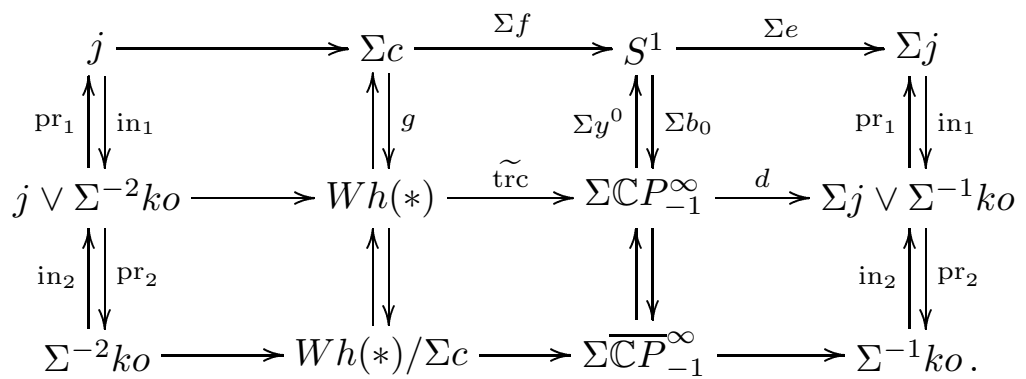

In particular there is a splitting

$$
W h(*) \simeq \Sigma c \vee(W h(*) / \Sigma c)
$$

where $W h(*) / \Sigma c$ is defined as the homotopy cofiber of $g$. 
Proof The composite $d \circ \Sigma b_{0}$ represents the generator of $\pi_{1}\left(\Sigma j \vee \Sigma^{-1} k o\right)$, hence factors as $\operatorname{in}_{1} \circ \Sigma e: S^{1} \rightarrow \Sigma j \rightarrow \Sigma j \vee \Sigma^{-1} k o$. We define $g: \Sigma c \rightarrow W h(*)$ as the induced map of homotopy fibers. It is well-defined up to homotopy since $\pi_{2}\left(\Sigma j \vee \Sigma^{-1} k o\right)=0$. This explains the downward cofiber sequences of the diagram.

To split $g$ we must show that $\operatorname{pr}_{1} \circ d$ factors as $\Sigma e \circ \Sigma y^{0}$, or equivalently that the composite

$$
\Sigma \overline{\mathbb{C} P}_{-1}^{\infty} \rightarrow \Sigma \mathbb{C} P_{-1}^{\infty} \stackrel{d}{\rightarrow} \Sigma j \vee \Sigma^{-1} k o \stackrel{\mathrm{pr}_{1}}{\longrightarrow} \Sigma j
$$

is null-homotopic. But this map lies in a zero group, because in the AtiyahHirzebruch spectral sequence

$$
E_{s, t}^{2}=H^{-s}\left(\Sigma \overline{\mathbb{C} P}_{-1}^{\infty} ; \pi_{t}(\Sigma j)\right) \Longrightarrow\left[\Sigma \overline{\mathbb{C} P}_{-1}^{\infty}, \Sigma j\right]_{s+t}
$$

all the groups $E_{s, t}^{2}$ with $s+t=0$ are zero.

Remark 3.7 Let $G / O$ be the homotopy fiber of the map of spaces $B O \rightarrow$ $B G$, and let Cok $J=\Omega^{\infty} c$ be the cokernel-of-J space. There is a (Sullivan) fiber sequence Cok $J \rightarrow G / O \rightarrow B S O$ [22, section 5C]. Waldhausen [48, 3.4] constructed a space level map $h w: G / O \rightarrow \Omega \Omega^{\infty} W h(*)$, using manifold models for $A(*)$. Hence there is a geometrically defined composite map Cok $J \rightarrow$ $G / O \rightarrow \Omega \Omega^{\infty} W h(*)$. Presumably this is homotopic to the infinite loop map $\Omega^{\infty} \Sigma^{-1} g$.

\section{A cofiber sequence}

We can analyze a variant of the lower cofiber sequence in 3.6 by passing to connective covers. There is a map of homotopy Cartesian squares from
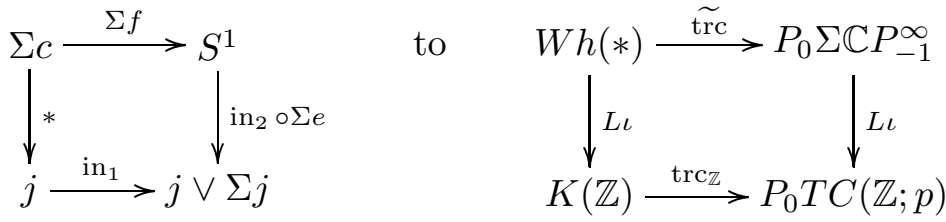

induced by $g, \Sigma b_{0}, \mathrm{in}_{1}$ and $\mathrm{in}_{1} \vee \mathrm{in}_{2}$ in the upper left, upper right, lower left and lower right corners, respectively. In the lower rows we are using the splittings $K(\mathbb{Z}) \simeq j \vee \Sigma^{5} k o$ and $P_{0} T C(\mathbb{Z} ; p) \simeq K\left(\mathbb{Z}_{p}\right) \simeq j \vee \Sigma j \vee \Sigma^{3} k u$ derived from 3.1. Let $\tau: W h(*) / \Sigma c \rightarrow P_{0} \Sigma \overline{\mathbb{C} P}_{-1}^{\infty}, \ell: W h(*) / \Sigma c \rightarrow \Sigma^{5} k o$ and $\ell: P_{0} \Sigma \overline{\mathbb{C} P}_{-1}^{\infty} \rightarrow$ $\Sigma^{3} k u$ be the cofiber maps induced by $\widetilde{\operatorname{trc}}: W h(*) \rightarrow P_{0} \Sigma \mathbb{C} P_{-1}^{\infty}, L \iota: W h(*) \rightarrow$ $K(\mathbb{Z})$ and $L \iota: P_{0} \Sigma \mathbb{C} P_{-1}^{\infty} \rightarrow P_{0} T C(\mathbb{Z} ; p)$, respectively. 
Theorem 3.8 Assume 3.2. There is a diagram of horizontal and vertical cofiber sequences:

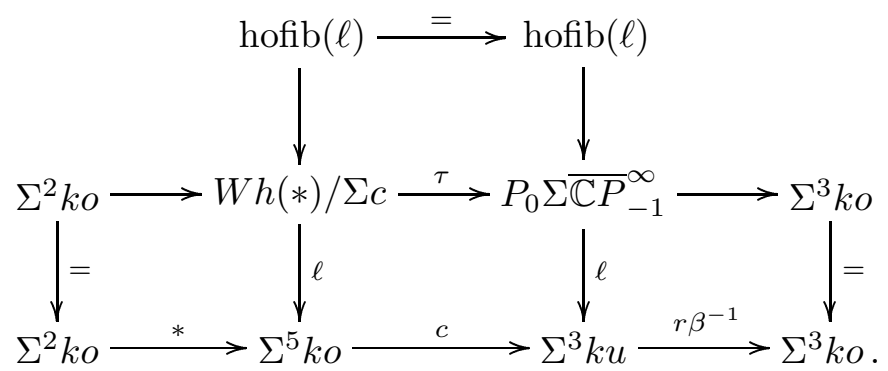

The map $\tau: W h(*) / \Sigma c \rightarrow P_{0} \Sigma \overline{\mathbb{C} P}_{-1}^{\infty}$ induces a split injection on homotopy groups in all degrees, and each map $\ell$ is $(2 p-3)$-connected. Thus

$$
\pi_{*}(\tau): \operatorname{tors} \pi_{*}(W h(*) / \Sigma c) \cong \operatorname{tors} \pi_{*}\left(\Sigma \overline{\mathbb{C} P}_{-1}^{\infty}\right) .
$$

Here tors $A$ denotes the torsion subgroup of an abelian group $A$.

Proof It follows from 3.1 and localization in algebraic $K$-theory that the map $\Sigma^{5} k o \rightarrow \Sigma^{3} k u$ induced by $\operatorname{trc}_{\mathbb{Z}}: K(\mathbb{Z}) \rightarrow P_{0} T C(\mathbb{Z} ; p) \simeq K\left(\mathbb{Z}_{p}\right)$ is the lift of $\Sigma c: \Sigma k o \rightarrow \Sigma k u$ to the 1-connected covers. This identifies the central homotopy Cartesian square in the diagram.

By comparing the vertical homotopy fibers in the last three homotopy Cartesian squares we obtain a cofiber sequence $c \vee \Sigma c \rightarrow \operatorname{hofib}(L) \rightarrow \operatorname{hofib}(\ell)$, as in [18, 3.6]. Hence each map $\ell$ is $(2 p-3)$-connected because $L$ is. There is a $(4 p-3)$ connected space level map from $S U$ to $\Omega^{\infty} \Sigma \overline{\mathbb{C} P}_{-1}^{\infty}$, as in $[18,(17)]$.

$$
B \phi: S U \rightarrow \Omega^{\infty} \Sigma \overline{\mathbb{C} P}_{-1}^{\infty} \stackrel{\Omega^{\infty} \ell}{\longrightarrow} S U .
$$

Its composite with $\Omega^{\infty} \ell$ to $\Omega^{\infty} \Sigma^{3} k u=S U$ loops to an H-map $\phi: B U \rightarrow B U$. Any such H-map is a series of Adams operations $\psi^{k}$, as in $[24,2.3]$, so $\pi_{*}(\phi ; \mathbb{Z} / p)$ only depends on $* \bmod q$ in positive degrees. Since $\ell$ is $(2 p-3)$-connected it follows that $\phi$ is $(2 p-4)$-connected, so $\pi_{*}(\phi ; \mathbb{Z} / p)$ is an isomorphism for $0<*<q$, and so $\pi_{*}(\phi)$ is an isomorphism for all $* \not \equiv 0 \bmod q$. Hence $\pi_{*}(\ell)$ is (split) surjective whenever $* \not \equiv 1 \bmod q$, cf. $[18,6.3(\mathrm{i})]$.

Finally $r \beta^{-1}$ is split surjective as a spectrum map, and $\pi_{*}\left(\Sigma^{3} k o\right)$ is zero for $* \equiv$ $1 \bmod q$, so $r \beta^{-1} \ell: P_{0} \Sigma \overline{\mathbb{C} P}_{-1}^{\infty} \rightarrow \Sigma^{3} k o$ induces a split surjection on homotopy in all degrees.

Remark 3.9 We still do not know the behavior of $\ell: W h(*) / \Sigma c \rightarrow \Sigma^{5} k o$ in degrees $* \equiv 1 \bmod q$. It induces the same homomorphism on homotopy as $\ell: P_{0} \Sigma \overline{\mathbb{C}}_{-1}^{\infty} \rightarrow \Sigma^{3} k u$, since $\pi_{*}(\tau)$ and $\pi_{*}(c)$ are isomorphisms in these degrees. 
Remark 3.10 By a result of Madsen and Schlichtkrull [23, 1.3] there is a splitting of implicitly $p$-completed spaces $\Omega^{\infty}\left(\Sigma \overline{\mathbb{C} P}_{-1}^{\infty}\right) \simeq Y \times S U$, where $\pi_{*}(Y) \cong$ tors $\pi_{*}\left(\Sigma \overline{\mathbb{C} P}_{-1}^{\infty}\right)$ is finite in each degree. The map

$$
Y \times S U \simeq \Omega^{\infty}\left(\Sigma \overline{\mathbb{C} P}_{-1}^{\infty}\right) \stackrel{\Omega^{\infty}\left(r \beta^{-1} \ell\right)}{\longrightarrow} \Omega^{\infty}\left(\Sigma^{3} k o\right) \simeq S p \simeq S O
$$

induces a split surjection on homotopy groups in all degrees, so the composite map $S U \stackrel{\mathrm{in}_{2}}{\longrightarrow} Y \times S U \rightarrow S O$ has homotopy fiber $B B O$, by real Bott periodicity. Hence there is a fiber sequence

$$
B B O \rightarrow \Omega^{\infty}(W h(*) / \Sigma c) \rightarrow Y
$$

and split short exact sequences

$$
0 \rightarrow \pi_{*}(B B O) \rightarrow \pi_{*}(W h(*) / \Sigma c) \rightarrow \pi_{*}(Y) \rightarrow 0
$$

in each degree.

\section{The suspended quaternionic projective spectrum}

After $p$-adic completion $\mathbb{C} P_{-1}^{\infty}$ splits as a wedge sum of $(p-1)$ eigenspectra $\mathbb{C} P_{-1}^{\infty}[a]$ for $-1 \leq a \leq p-3$, much like the $p$-complete (or $p$-local) Adams splitting of $k u$ from [1], and the $p$-complete splitting of $\Sigma^{\infty}\left(\mathbb{C} P_{+}^{\infty}\right)$ from $[25$, section 4.1]. Here $H^{*}\left(\mathbb{C} P_{-1}^{\infty}[a]\right) \cong \mathbb{Z}_{p}\left\{y^{k} \mid k \geq-1, k \equiv a \bmod p-1\right\}$, and similarly with mod $p$ coefficients.

Let $\mathbb{H} P^{\infty}$ be the infinite quaternionic projective spectrum. The "quaternionification" map $q: \mathbb{C} P_{-1}^{\infty} \rightarrow \mathbb{H} P_{+}^{\infty} \simeq S \vee \mathbb{H} P^{\infty}$ admits a (stable $p$-adic) section $s: \mathbb{H} P_{+}^{\infty} \rightarrow \mathbb{C} P_{-1}^{\infty}$. (It can be obtained by Thomifying the Becker-Gottlieb transfer map $\Sigma^{\infty}\left(B S_{+}^{3}\right) \rightarrow \Sigma^{\infty}\left(B S_{+}^{1}\right)$ associated to the sphere bundle $S^{2} \rightarrow$ $B S^{1} \rightarrow B S^{3}$, with respect to minus the tautological quaternionic line bundle over $B S^{3}=\mathbb{H} P^{\infty}$, and collapsing the bottom $(-4)$-cell. It is a section because the Euler characteristic $\chi\left(S^{2}\right)=2$ is a unit $\bmod p$.) This section $s$ identifies $S \vee \mathbb{H} P^{\infty}$ with the wedge sum of the even summands $\mathbb{C} P_{-1}^{\infty}[a]$ for $a=2 i$ with $0 \leq i \leq(p-3) / 2$.

Splitting off $S$, suspending once and passing to connected covers, we obtain maps $s^{\prime}: \Sigma \mathbb{H} P^{\infty} \rightarrow P_{0} \Sigma \overline{\mathbb{C} P}{ }_{-1}^{\infty}$ and $q^{\prime}: P_{0} \Sigma \overline{\mathbb{C} P}_{-1}^{\infty} \rightarrow \Sigma \mathbb{H} P^{\infty}$ whose composite is a $p$-adic equivalence.

Proposition 3.11 The map $s^{\prime}: \Sigma \mathbb{H} P^{\infty} \rightarrow P_{0} \Sigma \overline{\mathbb{C} P}_{-1}^{\infty}$ admits a lift

$$
\tilde{s}: \Sigma \mathbb{H} P^{\infty} \rightarrow W h(*) / \Sigma c
$$

over $\tau$, which is unique up to homotopy, and whose composite with

$$
q^{\prime} \circ \tau: W h(*) / \Sigma c \rightarrow \Sigma \mathbb{H} P^{\infty}
$$

is a $p$-adic equivalence. 
Proof The composite map $r \beta^{-1} \ell \circ s^{\prime}: \Sigma \mathbb{H} P^{\infty} \rightarrow \Sigma^{3} k o$ lies in a zero group, by the Atiyah-Hirzebruch spectral sequence

$$
E_{s, t}^{2}=H^{-s}\left(\Sigma \mathbb{H} P^{\infty} ; \pi_{t} \Sigma^{3} k o\right) \Longrightarrow\left[\Sigma \mathbb{H} P^{\infty}, \Sigma^{3} k o\right]_{s+t} .
$$

Hence $s^{\prime}$ admits a lift $\tilde{s}$, as claimed. In fact the lift is unique up to homotopy, since also $\left[\Sigma \mathbb{H} P^{\infty}, \Sigma^{3} k o\right]_{1}=0$.

\section{A second cofiber sequence}

We define $W h(*) /\left(\Sigma c, \Sigma \mathbb{H} P^{\infty}\right) \simeq \operatorname{hofib}\left(q^{\prime} \tau\right)$ as the homotopy cofiber of $\tilde{s}$, and write

$$
\frac{P_{0} \Sigma \overline{\mathbb{C}}_{-1}^{\infty}}{\Sigma \mathbb{H} P^{\infty}} \simeq P_{0} \Sigma \mathbb{C} P_{-1}^{\infty}[-1] \vee \bigvee_{i=1}^{(p-3) / 2} \Sigma \mathbb{C} P_{-1}^{\infty}[2 i-1]
$$

for the suspended homotopy cofiber of $s^{\prime}$. Then:

Theorem 3.13 Assume 3.2. There is a splitting

$$
W h(*) \simeq \Sigma c \vee \Sigma \mathbb{H} P^{\infty} \vee \frac{W h(*)}{\left(\Sigma c, \Sigma \mathbb{H} P^{\infty}\right)}
$$

and a cofiber sequence

$$
\Sigma^{2} k o \rightarrow \frac{W h(*)}{\left(\Sigma c, \Sigma \mathbb{H} P^{\infty}\right)} \stackrel{\tau}{\rightarrow} \frac{P_{0} \Sigma \overline{\mathbb{C} P}_{-1}^{\infty}}{\Sigma \mathbb{H} P^{\infty}} \stackrel{\delta}{\rightarrow} \Sigma^{3} k o .
$$

The map $\tau$ induces a split injection on homotopy groups in all degrees, and the map $\delta$ induces an injection on mod $p$ cohomology in degrees $\leq 2 p-3$. Thus

$$
\pi_{*}(W h(*)) \cong \pi_{*}(\Sigma c) \oplus \pi_{*}\left(\Sigma \mathbb{H} P^{\infty}\right) \oplus \operatorname{tors} \pi_{*}\left(\frac{\Sigma \overline{\mathbb{C} P}_{-1}^{\infty}}{\Sigma \mathbb{H} P^{\infty}}\right) .
$$

Proof The cofiber sequence arises by splitting off $\Sigma \mathbb{H} P^{\infty}$ from the middle horizontal cofiber sequence in 3.8. The assertion about $\tau$ follows by retraction from the corresponding statement in 3.8. The map $\delta$ is the composite of the maps

$$
\frac{P_{0} \Sigma \overline{\mathbb{C} P}_{-1}^{\infty}}{\Sigma \mathbb{H} P^{\infty}} \stackrel{\text { in }}{\longrightarrow} P_{0} \Sigma \overline{\mathbb{C} P}_{-1}^{\infty} \stackrel{\ell}{\longrightarrow} \Sigma^{3} k u \stackrel{r \beta^{-1}}{\longrightarrow} \Sigma^{3} k o .
$$

On mod $p$ cohomology $\left(r \beta^{-1}\right)^{*}$ is split injective and $\ell^{*}$ is injective in degrees $\leq 2 p-3$ by 3.8. The kernel of in* is $\Sigma H^{*}\left(\mathbb{H} P^{\infty} ; \mathbb{F}_{p}\right)$, which is concentrated in degrees $\equiv 1 \bmod 4$. But in degrees $\leq 2 p-3$ all of $H^{*}\left(\Sigma^{3} k o ; \mathbb{F}_{p}\right)$ is in degrees $\equiv 3 \bmod 4$, so also the composite $\delta^{*}$ is injective in this range of degrees.

Remark 3.14 Note that the upper cofiber sequence in 3.4 maps as in 3.6 to the middle horizontal cofiber sequence in 3.8 , which in turn maps to the cofiber sequence in 3.13. In 5.4 we will see that $\delta$ is $(4 p-2)$-connected. 


\section{Homotopical analysis}

\section{Homotopy of the fiber of the restricted $S^{1}$-transfer map}

To make the $p$-primary homotopy groups of $W h(*)$ explicit we refer to 3.8 and compute the $p$-torsion in the homotopy of $\overline{\mathbb{C} P}_{-1}^{\infty}$ in an initial range of degrees. This is related to $\mathbb{C} P^{\infty}$ by the cofiber sequence

$$
\Sigma \overline{\mathbb{C} P}_{-1}^{\infty} \rightarrow \Sigma \mathbb{C} P^{\infty} \stackrel{t}{\rightarrow} S
$$

extracted from (3.5). We also use the cofiber sequence

$$
c \wedge \mathbb{C} P^{\infty} \stackrel{f \wedge 1}{\longrightarrow} \mathbb{C} P^{\infty} \stackrel{e \wedge 1}{\longrightarrow} j \wedge \mathbb{C} P^{\infty}
$$

obtained by smashing (2.5) with $\mathbb{C} P^{\infty}$. There are Atiyah-Hirzebruch spectral sequences:

$$
\begin{aligned}
& E_{s, t}^{2}=H_{s}\left(\mathbb{C} P^{\infty} ; \pi_{t}(j)\right) \Longrightarrow j_{s+t}\left(\mathbb{C} P^{\infty}\right) \\
& E_{s, t}^{2}=H_{s}\left(\mathbb{C} P^{\infty} ; \pi_{t}(S)\right) \Longrightarrow \pi_{s+t}\left(\mathbb{C} P^{\infty}\right) \\
& E_{s, t}^{2}=H_{s}\left(\overline{\mathbb{C} P}_{-1}^{\infty} ; \pi_{t}(S)\right) \Longrightarrow \pi_{s+t}\left(\overline{\mathbb{C} P}_{-1}^{\infty}\right) .
\end{aligned}
$$

We will now account for the abutment of (4.2) in all degrees, and for (4.3) and (4.4) in total degrees $*<\left|\beta_{2} b_{1}\right|=(2 p+1) q$ and $*<\left|\beta_{2} b_{-1}\right|=(2 p+1) q-4$, respectively.

Let $v_{p}(n)$ be the $p$-adic valuation of a natural number $n$. In degrees $*<\left|\beta_{2}\right|=$ $(2 p+1) q-2$ the $p$-torsion in $\pi_{*}(S)=\pi_{*}^{S}$ is generated by the image-of-J classes $\bar{\alpha}_{i} \in \pi_{q i-1}^{S}$ of order $p^{1+v_{p}(i)}$ for $i \geq 1$, and the cokernel-of-J classes [37, 1.1.14]

$$
\beta_{1} \in \pi_{p q-2}^{S}, \quad \alpha_{1} \beta_{1} \in \pi_{(p+1) q-3}^{S}, \quad \beta_{1}^{2} \in \pi_{2 p q-4}^{S} \quad \text { and } \quad \alpha_{1} \beta_{1}^{2} \in \pi_{(2 p+1) q-5}^{S},
$$

each of order $p$.

Theorem 4.5 Above the horizontal axis and in total degrees $*<\left|\beta_{2}\right|-2$, the Atiyah-Hirzebruch $E_{s, t}^{\infty}$-term for $\pi_{*} \overline{\mathbb{C} P}_{-1}^{\infty}$ agrees with that for $j_{*}\left(\mathbb{C} P^{\infty}\right)$, plus the $\mathbb{Z} / p$-module generated by $\beta_{1} b_{m}, \alpha_{1} \beta_{1} b_{m p}, \beta_{1}^{2} b_{m}$ (and $\alpha_{1} \beta_{1}^{2} b_{m p}$, which is in a higher total degree) for $1 \leq m \leq p-3$, minus the $\mathbb{Z} / p$-module generated by $\alpha_{1} b_{m p}$ for $m \geq p-2$.

We give the proof in a couple of steps. 


\section{Connective J-theory of complex projective space}

On the horizontal axis the $E^{2}$-terms of (4.2) and (4.3) have the form $E_{*, 0}^{2}=$ $H_{*}\left(\mathbb{C} P^{\infty}\right)=\mathbb{Z}\left\{b_{n} \mid n \geq 1\right\}$, which has the structure of a divided power algebra on $b_{1}$. By Toda [43] or Mosher [31, 2.1], the corresponding part of the $E^{\infty}$-term of (4.3) consists of the polynomial algebra on $b_{1}$, i.e.,

$$
E_{2 n, 0}^{\infty}=\mathbb{Z}\left\{n ! b_{n}\right\} \subseteq E_{2 n, 0}^{2}=\mathbb{Z}\left\{b_{n}\right\}
$$

for all $n \geq 1$. Hence the order of the images of the differentials $d_{2 n, 0}^{r}$ landing in total degree $2 n-1$ all multiply to $n$ !.

It is known by [31, 4.7(a)] that these differentials from the horizontal axis land in the image-of-J, i.e., have the form $\theta b_{k}$ with $\theta$ a multiple of some $\bar{\alpha}_{i}$. Hence (4.6) also gives the $E^{\infty}$-term of (4.2) on the horizontal axis. Since the Atiyah-Hirzebruch spectral sequence for $j_{*}\left(\mathbb{C} P^{\infty}\right)$ only has classes in (even, odd) bidegrees above the horizontal axis, there can be no further differentials in (4.2). In even total degrees it follows that $j_{2 n}\left(\mathbb{C} P^{\infty}\right) \cong \mathbb{Z}\left\{n ! b_{n}\right\}$ for $n \geq 1$.

In odd total degrees, the $E^{2}$-term of (4.2) contains the classes $p^{e} \bar{\alpha}_{i} b_{k}$ in bidegree $(s, t)=(2 k, q i-1)$, for $0 \leq e \leq v_{p}(i)$. It follows that the $p$-valuation of the order of the groups $E_{s, t}^{2}$ in total degree $s+t=2 n-1$ equals $\sum_{e>0}\left[(n-1) / p^{e}(p-1)\right]$, so the $p$-valuation of the order of the finite group $j_{2 n-1}\left(\mathbb{C} P^{\infty}\right)$ is

$$
\sum_{e \geq 0}\left[\frac{n-1}{p^{e}(p-1)}\right]-\sum_{e \geq 0}\left[\frac{n}{p^{e} \cdot p}\right] .
$$

Here the second sum equals $v_{p}(n !)$. Compare $[18,4.3]$ due to Knapp. For $n \leq p^{2}(p-1)$ the terms with $e \geq 2$ vanish.

\section{Stable homotopy of complex projective space}

We now return to (4.3) where the $E^{2}$-term contains additional classes from $H_{*}\left(\mathbb{C} P^{\infty} ; \pi_{*}(c)\right)$. The primary operation $P^{1}$ detects $\alpha_{1}$, and $P^{1}\left(y^{k}\right)=k y^{k+p-1}$ in $\bmod p$ cohomology, so there are differentials $d^{q}\left(\theta b_{k+p-1}\right)=k \alpha_{1} \theta b_{k}$ for all $\theta \in \pi_{*}(S)$. In the case $\theta=1$ these differentials were already accounted for by the differentials leading to (4.6), but for $t<\left|\beta_{2}\right|$ there are also differentials

$$
d^{q}\left(\beta_{1} b_{k+p-1}\right)=\alpha_{1} \beta_{1} b_{k} \quad \text { and } \quad d^{q}\left(\beta_{1}^{2} b_{k+p-1}\right)=\alpha_{1} \beta_{1}^{2} b_{k}
$$

up to unit multiples, for $k \not \equiv 0 \bmod p, k \geq 1$. This leaves the classes $\alpha_{1} b_{m p}$ (already in $\left.j_{*}\left(\mathbb{C} P^{\infty}\right)\right), \alpha_{1} \beta_{1} b_{m p}$ and $\alpha_{1} \beta_{1}^{2} b_{m p}$ for $m \geq 1$ in odd total degrees, and the classes $\beta_{1} b_{1}, \ldots, \beta_{1} b_{p-2}, \beta_{1} b_{m p-1}$ for $m \geq 1, \beta_{1}^{2} b_{1}, \ldots, \beta_{1}^{2} b_{p-2}$ and $\beta_{1}^{2} b_{m p-1}$ for $m \geq 1$ in even total degrees. 
The (well-known) $p$-fold Toda bracket $\beta_{1}=\left\langle\alpha_{1}, \ldots, \alpha_{1}\right\rangle$ implies differentials

$$
d^{(p-1) q}\left(\theta \alpha_{1} b_{k+(p-1)^{2}}\right)=\theta \beta_{1} b_{k}
$$

when $k+(p-1)^{2}=m p$, up to unit multiples. So the classes $\alpha_{1} b_{m p}$ (from $\left.j_{*}\left(\mathbb{C} P^{\infty}\right)\right)$ and $\alpha_{1} \beta_{1} b_{m p}$ for $m \geq p-1$ support $d^{(p-1) q}$-differentials, which kill the classes $\beta_{1} b_{m p-1}$ and $\beta_{1}^{2} b_{m p-1}$ for $m \geq 1$. For bidegree reasons this accounts for all differentials in (4.3) in total degrees $*<\left|\beta_{2} b_{1}\right|$.

To pass from $\mathbb{C} P^{\infty}$ to $\overline{\mathbb{C P}}_{-1}^{\infty}$ we must take into account the differentials in (4.4) that cross the vertical axis, which amounts to the restricted $S^{1}$-transfer map $t$ as in (4.1). The image-of-J in its target $\pi_{*}(S)$ is hit by classes on the horizontal axis of $(4.3)$, by $[32,4.3]$ or Crabb and Knapp, cf. [18, 5.8]. The cokernel-of-J classes are hit by the differentials

$$
\begin{array}{rlrl}
d^{q}\left(\beta_{1} b_{p-2}\right) & =\alpha_{1} \beta_{1} b_{-1}, & d^{(p-1) q}\left(\alpha_{1} b_{(p-2) p}\right) & =\beta_{1} b_{-1}, \\
d^{q}\left(\beta_{1}^{2} b_{p-2}\right) & =\alpha_{1} \beta_{1}^{2} b_{-1}, \quad d^{(p-1) q}\left(\alpha_{1} \beta_{1} b_{(p-2) p}\right)=\beta_{1}^{2} b_{-1}
\end{array}
$$

in (4.4). Looking over the bookkeeping concludes the proof of Theorem 4.5.

\section{Torsion homotopy of the smooth Whitehead spectrum}

Theorem 4.7 (a) Assume 3.2. The torsion homotopy of $W h(*)$ decomposes as

$$
\operatorname{tors} \pi_{*}(W h(*)) \cong \pi_{*}(\Sigma c) \oplus \operatorname{tors} \pi_{*}\left(\Sigma \overline{\mathbb{C} P}_{-1}^{\infty}\right)
$$

in all degrees.

(b) In degrees $*<\left|\beta_{2}\right|+1=(2 p+1) q-1$

$$
\pi_{*}(\Sigma c) \cong \mathbb{Z} / p\left\{\Sigma \beta_{1}, \Sigma \alpha_{1} \beta_{1}, \Sigma \beta_{1}^{2}, \Sigma \alpha_{1} \beta_{1}^{2}\right\}
$$

with generators in degrees $p q-1,(p+1) q-2,2 p q-3$ and $(2 p+1) q-4$, respectively.

(c) In even degrees $*<\left|\beta_{2}\right|-1=(2 p+1) q-3$ the $p$-valuation of the order of tors $\pi_{2 n}\left(\Sigma \overline{\mathbb{C} P}_{-1}^{\infty}\right)$ equals

$$
\left(\left[\frac{n-1}{p-1}\right]+\left[\frac{n-1}{p(p-1)}\right]\right)-\left(\left[\frac{n}{p}\right]+\left[\frac{n}{p^{2}}\right]\right),
$$

plus 1 when $n=p^{2}-2+m p$ for $1 \leq m \leq p-3$, minus 1 when $n=p-1+m p$ for $m \geq p-2$.

(d) In odd degrees $*<\left|\beta_{2}\right|-1=(2 p+1) q-3$ the $p$-valuation of the order of tors $\pi_{2 n+1}\left(\Sigma \overline{\mathbb{C} P}_{-1}^{\infty}\right)$ equals 1 when $n=p^{2}-p-1+m$ or $n=2 p^{2}-2 p-2+m$ for $1 \leq m \leq p-3$, and is 0 otherwise. 
Example 4.8 (a) When $p=3$, the 3 -torsion in $\pi_{*} W h(*)$ has order 3 in degrees $11,16,18,20,21$ and 22 , order $3^{2}$ in degree 24 , order $3^{3}$ in degree 14 , and is trivial in the remaining degrees $*<25$.

(b) When $p=5$, the 5 -torsion in $\pi_{*} W h(*)$ has order 5 in degrees $18,26,28$, $34,36,39,41,43,48,50,52,54,58,60,62,64,68,70,72,77,78,79,80$ and 81 , order $5^{2}$ in degrees $42,44,56,74$ and 76 , order $5^{3}$ in degrees 46,66 and 82 , order $5^{4}$ in degree 84 , and is trivial in the remaining degrees $*<85$.

In roughly half this range we can give the following simpler statement.

Corollary 4.9 (a) For $p \geq 5$, the low-degree $p$-torsion in $\pi_{*} W h(*)$ is $\mathbb{Z} / p$ in degrees $*=2 n$ for $m(p-1)<n<m p$ and $1<m<p$, except in degree $2 p^{2}-$ $2 p-2$ (corresponding to $n=m p-1$ and $m=p-1$ ). The next $p$-torsion is $\mathbb{Z} / p\left\{\Sigma \beta_{1}\right\}$ in degree $2 p^{2}-2 p-1$, and a group of order $p^{2}$ in degree $2 p^{2}-2 p+2$.

(b) For $p=3$ the bottom 3 -torsion in $\pi_{*} W h(*)$ is $\mathbb{Z} / 3\left\{\Sigma \beta_{1}\right\}$ in degree 11, followed by $\mathbb{Z} / 3\left\{\Sigma \alpha_{1} \beta_{1}\right\} \oplus \mathbb{Z} / 9$ in degree 14 .

The asserted group structure of $\pi_{14} W h(*)_{(3)}$ can be obtained from 5.5(a) below and the mod 3 Adams spectral sequence.

Remark 4.10 Klein and the author showed in [18, 1.3(iii)] that for any odd prime $p$, regular or irregular, below degree $2 p^{2}-2 p-2$ there are direct summands $\mathbb{Z} / p$ in $\pi_{2 n} W h(*)$ for $m(p-1)<n<m p$ and $1<m<p$. The calculations above show that under the added hypothesis 3.2 , these classes constitute all of the $p$-torsion in $\pi_{*} W h(*)$, in this range of degrees.

\section{Cohomological analysis}

We can determine the mod $p$ cohomology of $W h(*)$ as a module over the Steenrod algebra $A$, up to an extension, in all degrees. To do this, we apply cohomology to the splitting and cofiber sequence in 3.13.

\section{Some cohomology modules}

Let us briefly write $H^{*}(X)=H^{*}\left(X ; \mathbb{F}_{p}\right)$ for the $\bmod p$ cohomology of a spectrum $X$, where $p$ is an odd prime. It is naturally a left module over the $\bmod p$ Steenrod algebra $A$ [40]. Let $A_{n}$ be the subalgebra of $A$ generated by the Bockstein operation $\beta$ and the Steenrod powers $P^{1}, \ldots, P^{p^{n-1}}$ and let $E_{n}$ be the exterior subalgebra generated by the Milnor primitives $\beta, Q_{1}, \ldots, Q_{n}$, where $Q_{0}=\beta$ and $Q_{n+1}=\left[P^{p^{n}}, Q_{n}\right]$. For an augmented subalgebra $B \subset A$ we write $I(B)=\operatorname{ker}\left(\epsilon: B \rightarrow \mathbb{F}_{p}\right)$ for the augmentation ideal, and let $A / / B=A \otimes_{B} \mathbb{F}_{p}=A / A \cdot I(B)$. 
Proposition 5.1 (a) $H^{*}(H \mathbb{Z}) \cong A / / E_{0}=A / A(\beta)$ and $H^{*}(\ell) \cong A / / E_{1}=$ $A / A\left(\beta, Q_{1}\right)$.

(b) The cofiber sequence $\Sigma^{q-1} \ell \rightarrow j \rightarrow \ell$ induces a nontrivial extension

$$
0 \rightarrow A / / A_{1} \rightarrow H^{*}(j) \rightarrow \Sigma^{p q-1} A / / A_{1} \rightarrow 0
$$

of $A$-modules. As an $A$-module $H^{*}(j)$ is generated by two classes 1 and $b$ in degree 0 and $p q-1$, respectively, with $\beta(b)=P^{p}(1)$.

(c) The cofiber sequence $S \stackrel{e}{\rightarrow} j \rightarrow \Sigma c$ induces an identification $H^{*}(\Sigma c) \cong$ $\operatorname{ker}\left(e^{*}: H^{*}(j) \rightarrow \mathbb{F}_{p}\right)$. There is a nontrivial extension

$$
0 \rightarrow I(A) / A\left(\beta, P^{1}\right) \rightarrow H^{*}(\Sigma c) \rightarrow \Sigma^{p q-1} A / / A_{1} \rightarrow 0
$$

of $A$-modules.

Proof For (a), see [2, 2.1]. For (c), clearly the given cofiber sequence identifies $H^{*}(\Sigma c)$ with the positive degree part of $H^{*}(j)$. The long exact sequence in cohomology associated to the cofiber sequence given in (b) is:

$$
\Sigma^{q} A / / E_{1} \stackrel{\left(\psi^{r}-1\right)^{*}}{\longrightarrow} A / / E_{1} \rightarrow H^{*}(j) \rightarrow \Sigma^{q-1} A / / E_{1} \stackrel{\left(\psi^{r}-1\right)^{*}}{\longrightarrow} \Sigma^{-1} A / / E_{1} .
$$

The map $e: S \rightarrow j$ is $(p q-2)$-connected [37, 1.1.14], so $e^{*}: H^{*}(j) \rightarrow H^{*}(S)=$ $\mathbb{F}_{p}$ is an isomorphism for $* \leq p q-2$. Thus $P^{1} \in A / / E_{1}$ is in the image of $\left(\psi^{r}-1\right)^{*}$, and so $\left(\psi^{r}-1\right)^{*}$ is induced up over $A_{1} \subset A$ by

$$
\Sigma^{q} A_{1} / / E_{1} \stackrel{P^{1}}{\longrightarrow} A_{1} / / E_{1},
$$

which has kernel $\Sigma^{p q} \mathbb{F}_{p}$ generated by $\Sigma^{q} P^{p-1}$ and cokernel $\mathbb{F}_{p}$ generated by 1. Hence there is an extension $A / / A_{1} \rightarrow H^{*}(j) \rightarrow \Sigma^{p q-1} A / / A_{1}$. Note that the bottom classes in $A / / A_{1}$ are 1 and $P^{p}$ in degrees 0 and $p q$, respectively. Let $b \in H^{p q-1}(j)$ be the class mapped to $\Sigma^{p q-1}(1)$ in $\Sigma^{p q-1} A / / A_{1}$. By the Hurewicz theorem for $\Sigma c$ it is dual to the Hurewicz image of the bottom class $\Sigma \beta_{1} \in \pi_{p q-1}(\Sigma c)$. Since $\beta_{1} \in \pi_{p q-2}(c) \subset \pi_{p q-2}(S)$ has order $p$ there is a nontrivial Bockstein $\beta(b)$ in $H^{*}(\Sigma c)$, and thus also in $H^{*}(j)$. The only possible value in degree $p q$ is $P^{p}(1)$. Part (c) now follows easily from (b).

Proposition 5.2 (a) $H^{*}\left(\Sigma \mathbb{H} P^{\infty}\right) \cong \mathbb{F}_{p}\left\{\Sigma y^{k} \mid k \geq 2\right.$ even $\}$.

(b) $H^{*}\left(\Sigma \mathbb{C} P_{-1}^{\infty}[-1]\right) \cong \Sigma^{-1} A / C$. Here $C \subset A$ is the annihilator ideal of $\Sigma y^{-1}$, which is spanned over $\mathbb{F}_{p}$ by all admissible monomials in $A$ except 1 and the $P^{i}$ for $i \geq 1$.

(c) The cofiber sequence $P_{0} \Sigma \mathbb{C} P_{-1}^{\infty}[-1] \rightarrow \Sigma \mathbb{C} P_{-1}^{\infty}[-1] \rightarrow \Sigma^{-1} H \mathbb{Z}$ induces an identification $H^{*}\left(P_{0} \Sigma \mathbb{C} P_{-1}^{\infty}[-1]\right) \cong \Sigma^{-2} C / A(\beta)$.

(d) For $1 \leq i \leq(p-3) / 2$ there are isomorphisms $H^{*}\left(\Sigma \mathbb{C} P_{-1}^{\infty}[2 i-1]\right) \cong$ $\mathbb{F}_{p}\left\{\Sigma y^{k} \mid k=2 i-1+m(p-1), m \geq 0\right\}$. 
Proof Any admissible monomial $P^{I}$ with $I=\left(i_{1}, \ldots, i_{n}\right)$ and $n \geq 2$ acts trivially on $\Sigma y^{-1}$ because $z=P^{i_{n}}\left(\Sigma y^{-1}\right)$ is in the image from $H^{*}\left(\Sigma \mathbb{C} P^{\infty}\right)$, which is an unstable $A$-module, and then $P^{i_{n-1}}(z)=0$ by instability.

\section{Cohomology of the smooth Whitehead spectrum}

Proposition 5.3 The A-module homomorphism

$$
\delta^{*}: H^{*}\left(\Sigma^{3} k o\right) \rightarrow H^{*}\left(P_{0} \Sigma \overline{\mathbb{C} P}_{-1}^{\infty} / \Sigma \mathbb{H} P^{\infty}\right)
$$

splits as the direct sum of the injection

$$
\Sigma^{q-1} A / / E_{1} \rightarrow \Sigma^{-2} C / A(\beta)
$$

taking $\Sigma^{q-1}(1)$ to $\Sigma^{-2} Q_{1}$, and the homomorphisms

$$
\begin{aligned}
\delta_{i}^{*}: \Sigma^{4 i-1} A / / E_{1} & \rightarrow H^{*}\left(\Sigma \mathbb{C} P_{-1}^{\infty}[2 i-1]\right) \\
& \cong \mathbb{F}_{p}\left\{\Sigma y^{k} \mid k=2 i-1+m(p-1), m \geq 0\right\}
\end{aligned}
$$

taking $\Sigma^{4 i-1}(1)$ to $\Sigma y^{2 i-1}$ for $1 \leq i \leq(p-3) / 2$.

Proof By (2.4) and 5.1(a) the source of $\delta^{*}$ splits as the direct sum of the cyclic $A$-modules $\Sigma^{4 i-1} A / / E_{1}$ for $1 \leq i \leq(p-1) / 2$. Here $4 i-1=q-1$ for $i=(p-1) / 2$. Hence $\delta^{*}$ is determined as an $A$-module homomorphism by its value on the generators $\Sigma^{4 i-1}(1)$. These are all in degrees $\leq q-1=2 p-3$, and $\delta^{*}$ is injective in this range by 3.13. By (3.12), 5.2(c) and (d) the target of $\delta^{*}$ splits as the direct sum of $\mathbb{F}_{p}\left\{\Sigma y^{k} \mid k \equiv 2 i-1+m(p-1), m \geq 0\right\}$ for $1 \leq i \leq(p-3) / 2$ and $\Sigma^{-2} C / A(\beta)$. The bottom class of the latter is $\Sigma^{-2} Q_{1}$, in degree $q-1$. Hence the target of $\delta^{*}$ has rank 1 in each degree $4 i-1$ for $1 \leq i \leq(p-1) / 2$, and so (up to a unit which we suppress) $\delta^{*}$ maps $\Sigma^{4 i-1}(1)$ to $\Sigma y^{2 i-1}$ for $1 \leq i \leq(p-3) / 2$ and $\Sigma^{q-1}(1)$ to $\Sigma^{-2} Q_{1}$.

The homomorphism $\Sigma^{q-1} A / / E_{1} \rightarrow \Sigma^{-2} C / A(\beta)$ is injective, as its continuation into $\Sigma^{-2} A / / E_{0}$ is induced up over $E_{1} \subset A$ from the injection $\Sigma^{q-1} \mathbb{F}_{p} \rightarrow$ $\Sigma^{-2} E_{1} / / E_{0}$ taking $\Sigma^{q-1}(1)$ to $\Sigma^{-2} Q_{1}$.

Theorem 5.4 Assume 3.2. There is a splitting

$$
H^{*}(W h(*)) \cong H^{*}(\Sigma c) \oplus H^{*}\left(\Sigma \mathbb{H} P^{\infty}\right) \oplus H^{*}\left(\frac{W h(*)}{\left(\Sigma c, \Sigma \mathbb{H} P^{\infty}\right)}\right)
$$

and an extension of $A$-modules

$$
0 \rightarrow \operatorname{cok}\left(\delta^{*}\right) \rightarrow H^{*}\left(\frac{W h(*)}{\left(\Sigma c, \Sigma \mathbb{H} P^{\infty}\right)}\right) \rightarrow \Sigma^{-1} \operatorname{ker}\left(\delta^{*}\right) \rightarrow 0
$$


where

$$
\operatorname{cok}\left(\delta^{*}\right) \cong \Sigma^{-2} C / A\left(\beta, Q_{1}\right) \oplus \bigoplus_{i=1}^{(p-3) / 2} H^{*}\left(\Sigma \mathbb{C} P_{-1}^{\infty}[a]\right) / A\left(\Sigma y^{a}\right)
$$

and

$$
\Sigma^{-1} \operatorname{ker}\left(\delta^{*}\right) \cong \bigoplus_{i=1}^{(p-3) / 2} \Sigma^{2 a} C_{a} / A\left(\beta, Q_{1}\right) .
$$

In both sums we briefly write $a=2 i-1$, so $a$ is odd with $1 \leq a \leq p-4$. Here $H^{*}\left(\Sigma \mathbb{C} P_{-1}^{\infty}[a]\right)=\mathbb{F}_{p}\left\{\Sigma y^{k} \mid k \equiv a \bmod p-1, k \geq a\right\}, A\left(\Sigma y^{a}\right) \subset H^{*}\left(\Sigma \mathbb{C} P_{-1}^{\infty}[a]\right)$ is the submodule generated by $\Sigma y^{a}$, and $C_{a} \subset A$ is the annihilator ideal of $\Sigma y^{a} \in H^{*}\left(\Sigma \mathbb{C} P_{-1}^{\infty}[a]\right)$.

Proof The splitting and extension follow by applying cohomology to 3.13. The cohomologies of $\Sigma c$ and $\Sigma \mathbb{H} P^{\infty}$ are given in 5.1(c) and 5.2(a), respectively. The descriptions of $\operatorname{ker}\left(\delta^{*}\right)$ and $\operatorname{cok}\left(\delta^{*}\right)$ are immediate from 5.3.

Example 5.5 (a) When $p=3$ there is a splitting

$$
H^{*}(W h(*)) \cong H^{*}(\Sigma c) \oplus H^{*}\left(\Sigma \mathbb{H} P^{\infty}\right) \oplus \Sigma^{-2} C / A\left(\beta, Q_{1}\right) .
$$

(b) When $p=5$ there is an extension

$$
\begin{aligned}
0 \rightarrow \Sigma^{-2} C / A\left(\beta, Q_{1}\right) \oplus H^{*} & \left(\Sigma \mathbb{C} P_{-1}^{\infty}[1]\right) / A(\Sigma y) \\
& \rightarrow H^{*}\left(\frac{W h(*)}{\left(\Sigma c, \Sigma \mathbb{H} P^{\infty}\right)}\right) \rightarrow \Sigma^{2} C_{1} / A\left(\beta, Q_{1}\right) \rightarrow 0
\end{aligned}
$$

where

$$
H^{*}\left(\Sigma \mathbb{C} P_{-1}^{\infty}[1]\right) / A(\Sigma y) \cong \mathbb{F}_{p}\left\{\Sigma y^{k} \mid k \equiv 1 \bmod p-1, k \geq 1 ; k \neq p^{e}, e \geq 0\right\}
$$

and $C_{1} \subset A$ is spanned over $\mathbb{F}_{p}$ by all admissible monomials in $A$ except 1 and the $P^{I}$ for $I=\left(p^{e}, p^{e-1}, \ldots, p, 1\right)$ with $e \geq 0$.

Remark 5.6 (a) The $A$-module $\Sigma^{-2} C / A\left(\beta, Q_{1}\right)$ can be shown to split off from $H^{*}\left(W h(*) /\left(\Sigma c, \Sigma \mathbb{H} P^{\infty}\right)\right)$ by considering the lower cofiber sequence in 3.6.

(b) For $p \geq 5$ the extension of $\Sigma^{2} C_{1} / A\left(\beta, Q_{1}\right)$ by $H^{*}\left(\Sigma \mathbb{C} P_{-1}^{\infty}[1]\right) / A(\Sigma y)$ is not split. By 4.9 the bottom $p$-torsion homotopy of $W h(*)$ is $\mathbb{Z} / p$ in degree $4 p-2$, which implies that there is a nontrivial mod $p$ Bockstein relating the bottom classes $\Sigma^{2} P^{2}$ and $\Sigma y^{2 p-1}$ of these two $A$-modules, respectively. 


\section{Applications to automorphism spaces}

We now recall the relation between Whitehead spectra, smooth concordance spaces and diffeomorphism groups, to allow us to formulate a geometric interpretation of our calculations.

\section{Spaces of concordances and $h$-cobordisms}

Let $M$ be a compact smooth $n$-manifold, possibly with corners, and let $I=$ $[0,1]$ be the unit interval. To study the automorphism space $\operatorname{DIFF}(M)$ of self-diffeomorphisms of $M$ relative to the boundary $\partial M$, one is led to study the concordance space

$$
C(M)=\operatorname{DIFF}(M \times I, M \times 1)
$$

of smooth concordances on $M$, also known as the pseudo-isotopy space of $M$ [17]. This equals the space of self-diffeomorphisms $\psi$ of the cylinder $M \times I$ relative to the part $\partial M \times I \cup M \times 0$ of the boundary. Both $D I F F(M)$ and $C(M)$ can be viewed as topological or simplicial groups, and there is a fiber sequence

$$
\operatorname{DIFF}(M \times I) \rightarrow C(M) \stackrel{r}{\rightarrow} \operatorname{DIFF}(M)
$$

where $r$ restricts a concordance $\psi$ to the upper end $M \times 1$ of the cylinder.

Let $J=[0, \infty)$. The smooth $h$-cobordism space $H(M)$ of $M[48$, section 1$]$ is the space of smooth codimension zero submanifolds $W \subset M \times J$ that are $h$-cobordisms with $M=M \times 0$ at one end, relative to the trivial $h$-cobordism $\partial M \times I$. There is a fibration over $H(M)$ with $C(M)$ as fiber and the contractible space of collars on $M \times 0$ in $M \times J$ as total space. Hence $H(M)$ is a non-connective delooping of $C(M)$, i.e., $C(M) \simeq \Omega H(M)$. The homotopy types of the diffeomorphism group $\operatorname{DIFF}(M)$, the concordance space $C(M)$ and the $h$-cobordism space $H(M)$ are of intrinsic interest in geometric topology.

There are stabilization maps $\sigma: C(M) \rightarrow C(I \times M)$ and $\sigma: H(M) \rightarrow H(I \times M)$. By Igusa's stability theorem [17], the former map is at least $k$-connected when $n \geq \max \{2 k+7,3 k+4\}$. Then this is also a lower bound for the connectivity of the canonical map

$$
\Sigma: C(M) \rightarrow \mathcal{C}(M)=\operatorname{hocolim}_{\ell} C\left(I^{\ell} \times M\right)
$$

to the mapping telescope of the stabilization map $\sigma$ repeated infinitely often. We call $\mathcal{C}(M)$ the stable concordance space of $M$, and call the connectivity of $\Sigma: C(M) \rightarrow \mathrm{C}(M)$ the concordance stable range of $M$. Likewise there is a stable $h$-cobordism space $\mathcal{H}(M)=\operatorname{hocolim}_{\ell} H\left(I^{\ell} \times M\right)$, and $\mathcal{C}(M) \simeq \Omega \mathcal{H}(M)$. The connectivity of the map $H(M) \rightarrow \mathcal{H}(M)$ is one more than the concordance stable range of $M$. 


\section{The stable parametrized $h$-cobordism theorem}

Waldhausen proved in [51] that when $X=M$ is a compact smooth manifold there is a homotopy equivalence

$$
\mathcal{H}(M) \simeq \Omega \Omega^{\infty} W h(M),
$$

i.e., that the Whitehead space $\Omega^{\infty} W h(M)$ of $M$ is a delooping of the stable $h$ cobordism space $\mathcal{H}(M)$ of $M$. This stable parametrized $h$-cobordism theorem is the fundamental result linking algebraic $K$-theory of spaces to concordance theory. At the level of $\pi_{0}$ it recovers the (stable) $h$ - and $s$-cobordism theorems of Smale, Barden, Mazur and Stallings. Waldhausen's theorem includes in particular the assertion that the stable $h$-cobordism space $\mathcal{H}(M)$ and the stable concordance space $\mathcal{C}(M)$ are infinite loop spaces.

The functor $X \mapsto A(X)$ preserves connectivity of mappings, in the sense that if $X \rightarrow Y$ is a $k$-connected map with $k \geq 2$ then $A(X) \rightarrow A(Y)$ is also $k$ connected [46, 1.1], [6, 10.9]. It follows that $W h(M), \mathcal{H}(M)$ and $\mathcal{C}(M)$ take $k$-connected maps to $k$-, $(k-1)$ - and $(k-2)$-connected maps, respectively, for $k \geq 2$.

Let $\pi=\pi_{1}(M)$ be the fundamental group of $X=M$. The classifying map $M \rightarrow B \pi$ for the universal covering of $M$ is $k$-connected for some $k \geq 2$, so also $A(M) \rightarrow A(B \pi)$ is $k$-connected. Let $R=\mathbb{Z}[\pi]$. Then the linearization map $L: A(B \pi) \rightarrow K(R)$ is a rational equivalence by [46, 2.2]. Hence rational information about $K(R)$ gives rational information about $A(M)$ up to degree $k$, and about $\mathrm{C}(M)$ up to degree $k-2$, which in turn agrees with $C(M)$ in the concordance stable range.

For example, Farrell and Hsiang [14] show that $\pi_{m} C\left(D^{n}\right) \otimes \mathbb{Q}$ has rank 1 in all degrees $m \equiv 3 \bmod 4$, and rank 0 in other degrees, for $n$ sufficiently large with respect to $m$. From this they deduce that $\pi_{m} D I F F\left(D^{n}\right) \otimes \mathbb{Q}$ has rank 1 for $m \equiv 3 \bmod 4$ and $n$ odd, and rank 0 otherwise, always assuming that $m$ is in the concordance stable range for $D^{n}$.

For $\pi$ a finite group, $A(X)$ and $W h(X)$ are of finite type by theorems of Dwyer [10] and Betley [3], so the integral homotopy type is determined by the rational homotopy type and the $p$-adic homotopy type for all primes $p$. Therefore our results on the $p$-adic homotopy type of $W h(*)$ have following application:

Theorem 6.3 Assume 3.2.

(a) Suppose $p \geq 5$ and let $M$ be a $(4 p-2)$-connected compact smooth manifold whose concordance stable range exceeds $(4 p-4)$, e.g., an $n$-manifold with 
$n \geq 12 p-5$. Then the first $p$-torsion in the homotopy of the smooth concordance space $C(M)$, and in the homotopy of the smooth $h$-cobordism space $H(M)$, is

$$
\pi_{4 p-4} C(M)_{(p)} \cong \pi_{4 p-3} H(M)_{(p)} \cong \mathbb{Z} / p .
$$

(b) Suppose $p=3$ and let $M$ be an 11-connected compact smooth manifold whose concordance stable range exceeds 9 , e.g., an $n$-manifold with $n \geq 34$. Then the first 3 -torsion in the homotopy of the smooth concordance space $C(M)$, and in the homotopy of the smooth $h$-cobordism space $H(M)$, is

$$
\pi_{9} C(M)_{(3)} \cong \pi_{10} H(M)_{(3)} \cong \mathbb{Z} / 3 .
$$

Proof The first $p$-torsion in $\pi_{*} W h(*)$ is $\mathbb{Z} / p$ in degree $*=4 p-2$ for $p \geq 5$, and $\mathbb{Z} / 3\left\{\Sigma \beta_{1}\right\}$ in degree $*=11$ for $p=3$, and $\pi_{*} W h(*)$ is finite in all of these degrees. When $M$ is $(4 p-2)$-connected, resp. 11-connected, the map $\pi_{*} W h(M) \rightarrow \pi_{*} W h(*)$ is an isomorphism in this degree. And $\pi_{*-2} \mathrm{C}(M) \cong$ $\pi_{*-1} \mathcal{H}(M) \cong \pi_{*} W h(M)$. So if the concordance stable range is at least $(4 p-3)$, resp. 10 , also $\pi_{*-2} C(M) \cong \pi_{*-2} \mathrm{C}(M)$ and $\pi_{*-1} H(M) \cong \pi_{*-1} \mathcal{H}(M)$ in this degree.

Similar statements may of course be given for when the subsequent torsion groups in $\pi_{*} W h(*)$ agree with $\pi_{*-2} C(M)$ and $\pi_{*-1} H(M)$, under stronger connectivity and dimension hypotheses.

By $[18,1.4]$ there is a summand $\mathbb{Z} / p$ in $\pi_{4 p-4} C(M)$ for any $p \geq 5$, regular or not, but we need 3.2 to show that this is the first $p$-torsion in $\pi_{*} C(M)$.

Theorem 6.4 Assume 3.2.

(a) Suppose $p \geq 5$ and let $M=D^{n}$ with $n \geq 12 p-5$. Then $\pi_{4 p-4} D I F F\left(D^{n+1}\right)$ or $\pi_{4 p-4} D I F F\left(D^{n}\right)$ contains an element of order $p$.

(b) Suppose $p=3$ and let $M=D^{n}$ with $n \geq 34$. Then $\pi_{9} D I F F\left(D^{n+1}\right)$ or $\pi_{9} \operatorname{DIFF}\left(D^{n}\right)$ contains an element of order 3 .

Proof Consider the exact sequence in homotopy induced by (6.1), with $D^{n} \times$ $I \cong D^{n+1}$. A $\mathbb{Z} / p$ in $\pi_{m} C\left(D^{n}\right)$ either comes from $\pi_{m} D I F F\left(D^{n+1}\right)$, which known to be finite in these cases by [14], or maps to $\pi_{m} D I F F\left(D^{n}\right)$. 


\section{References}

[1] J F Adams, Lectures on generalised cohomology, from: "Category Theory, Homology Theory and their Applications", Proc. Conf. Seattle Res. Center Battelle Mem. Inst. 1968, 3 (1969) 1-138

[2] J F Adams, S B Priddy, Uniqueness of BSO, Math. Proc. Cambridge Philos. Soc. 80 (1976) 475-509

[3] S Betley, On the homotopy groups of $A(X)$, Proc. Amer. Math. Soc. 98 (1986) 495-498

[4] S Bloch, K Kato, p-adic étale cohomology, Publ. Math. Inst. Hautes Étud. Sci. 63 (1986) 107-152

[5] M Bökstedt, W-C Hsiang, I Madsen, The cyclotomic trace and algebraic $K$-theory of spaces, Invent. Math. 111 (1993) 865-940

[6] M Bökstedt, I Madsen, Topological cyclic homology of the integers, Asterisque 226 (1994) $57-143$

[7] M Bökstedt, I Madsen, Algebraic K-theory of local number fields: the unramified case, from: "Prospects in topology, Princeton, NJ, 1994", Ann. of Math. Studies 138, Princeton University Press (1995) 28-57

[8] A K Bousfield, The localization of spectra with respect to homology, Topology 18 (1979) 257-281

[9] B I Dundas, Relative $K$-theory and topological cyclic homology, Acta Math. 179 (1997) 223-242

[10] W G Dwyer, Twisted homological stability for general linear groups, Ann. of Math. 111 (1980) 239-251

[11] W G Dwyer, E M Friedlander, Algebraic and étale $K$-theory, Trans. AMS 292 (1985) 247-280

[12] W G Dwyer, E M Friedlander, Topological models for arithmetic, Topology 33 (1994) 1-24

[13] W G Dwyer, S A Mitchell, On the $K$-theory spectrum of a ring of algebraic integers, $K$-Theory 14 (1998) 201-263

[14] F T Farrell, W C Hsiang, On the rational homotopy groups of the diffeomorphism groups of discs, spheres and aspherical manifolds, from: "Algebr. geom. Topol. Stanford/Calif. 1976", Proc. Symp. Pure Math. 32, Part 1 (1978) 325337

[15] L Hesselholt, I Madsen, On the $K$-theory of finite algebras over Witt vectors of perfect fields, Topology 36 (1997) 29-101

[16] R T Hoobler, When is $\operatorname{Br}(X)=\operatorname{Br}^{\prime}(X)$ ?, from: "Brauer groups in ring theory and algebraic geometry, Proc. Antwerp 1981", from: "Lecture Notes in Math.", 917 Springer-Verlag (1982) 231-244 
[17] K Igusa, The stability theorem for smooth pseudoisotopies, K-Theory 2 (1988) $1-355$

[18] J.R Klein, J Rognes, The fiber of the linearization map $A(*) \rightarrow K(\mathbb{Z})$, Topology 36 (1997) 829-848

[19] K-H Knapp, Im $(J)$-theory for torsion-free spaces The complex projective space as an example, Revised version of Habilitationsschrift Bonn 1979, in preparation

[20] S Lichtenbaum, On the values of zeta and L-functions, I, Annals of Math. 96 (1972) 338-360

[21] S Lichtenbaum, Values of zeta functions, étale cohomology, and algebraic $K$ theory, from: "Algebraic $K$-theory, II: "Classical" algebraic $K$-theory and connections with arithmetic", Lecture Notes in Math. 342 Springer-Verlag (1973) 489-501

[22] I Madsen, RJ Milgram, The classifying spaces for surgery and cobordism of manifolds, Annals of Mathematics Studies 92, Princeton University Press (1979)

[23] I Madsen, C Schlichtkrull, The circle transfer and $K$-theory, (Grove, Karsten et al Editors)from: "Geometry and topology, Aarhus Proceedings of the conference on geometry and topology, Aarhus, Denmark, August 10-16, 1998", Contemp. Math. 258 American Mathematical Society (2000) 307-328

[24] I Madsen, V Snaith, J Tornehave, Infinite loop maps in geometric topology, Math. Proc. Camb. Phil. Soc. 81 (1977) 399-429

[25] I Madsen, U Tillmann, The stable mapping class group and $Q\left(\mathbb{C} P_{+}^{\infty}\right)$, Invent. Math. 145 (2001) 509-544

[26] J P May, F Quinn, N Ray, with contributions by J Tornehave, $E_{\infty}$ ring spaces and $E_{\infty}$ ring spectra, Lecture Notes in Math. 577 Springer-Verlag (1977)

[27] J S Milne, Étale cohomology, Princeton Mathematical Series 33, Princeton University Press (1980)

[28] J S Milne, Arithmetic duality theorems, Perspectives in Mathematics 1 Academic Press, Inc. (1986)

[29] G Mislin, Localization with respect to K-theory, J. Pure Appl. Algebra 10 (1977/78) 201-213

[30] S A Mitchell, On p-adic topological $K$-theory, (P G Goerss et al Editors)from: "Algebraic $K$-theory and algebraic topology, Proceedings of the NATO Advanced Study Institute, Lake Louise, Alberta, Canada, December 12-16, 1991", NATO ASI Ser. Ser. C, Math. Phys. Sci. 407 Kluwer Academic Publishers (1993) 197-204

[31] R E Mosher, Some stable homotopy of complex projective space, Topology 7 (1968) 179-193 
[32] J Mukai, The $S^{1}$-transfer map and homotopy groups of suspended complex projective spaces, Math. J. Okayama Univ. 24 (1982) 179-200

[33] D Quillen, Higher algebraic $K$-theory. I, from: "Algebraic $K$-Theory, I: Higher $K$-theories", Lecture Notes Math. 341 Springer-Verlag (1973) 85-147

[34] D Quillen, Finite generation of the groups $K_{i}$ of rings of algebraic integers, from: "Algebraic $K$-Theory, I: Higher $K$-theories", Lecture Notes in Math. 341 Springer-Verlag (1973) 179-198

[35] D Quillen, Higher algebraic K-theory, from: "Proc. Intern. Congress Math. Vancouver, 1974", I Canad. Math. Soc. (1975) 171-176

[36] D Quillen, Letter from Quillen to Milnor on $\operatorname{Im}\left(\pi_{i} O \rightarrow \pi_{i}^{s} \rightarrow K_{i} \mathbb{Z}\right)$, from: "Algebraic $K$-theory, Proc. Conf. Northwestern Univ. Evanston, Ill. 1976", Lecture Notes in Math. 551 Springer-Verlag (1976) 182-188

[37] D C Ravenel, Complex cobordism and stable homotopy groups of spheres, Pure and Applied Math. 121 Academic Press (1986)

[38] J Rognes, Two-primary algebraic $K$-theory of pointed spaces, Topology 41 (2002) 873-926

[39] J Rognes, C Weibel, Two-primary algebraic $K$-theory of rings of integers in number fields, J. Am. Math. Soc. 13 (2000) 1-54

[40] N E Steenrod, Cohomology operations, Annals of Mathematics Studies 50, Princeton University Press (1962)

[41] A Suslin, V Voevodsky, Bloch-Kato conjecture and motivic cohomology with finite coefficients, (B B Gordon et al Editors)from: "The arithmetic and geometry of algebraic cycles", NATO ASI Ser. Ser. C, Math. Phys. Sci. 548 Kluwer Academic Publishers (2000) 117-189

[42] J Tate, Duality theorems in Galois cohomology over number fields, from: "Proc. Intern. Congress Math. Stockholm, 1962", Inst. Mittag-Leffler (1963) 234-241

[43] H Toda, A topological proof of theorems of Bott and Borel-Hirzebruch for homotopy groups of unitary groups, Mem. Coll. Sci. Univ. Kyoto, Ser. A 32 (1959) 103-119

[44] V Voevodsky, The Milnor Conjecture, preprint (1996)

[45] V Voevodsky, On 2-torsion in motivic cohomology, preprint (2001)

[46] F Waldhausen, Algebraic $K$-theory of topological spaces. I, from: "Algebr. geom. Topol. Stanford/Calif. 1976", Proc. Symp. Pure Math. 32, Part 1 (1978) $35-60$

[47] F Waldhausen, Algebraic K-theory of topological spaces, II, from: "Algebraic topology, Proc. Symp. Aarhus 1978", Lecture Notes in Math. 763 SpringerVerlag (1979) 356-394

[48] F Waldhausen, Algebraic $K$-theory of spaces, a manifold approach, from: "Current trends in algebraic topology, Semin. London/Ont. 1981", CMS Conf. Proc. 2, Part 1 (1982) 141-184 
[49] F Waldhausen, Algebraic $K$-theory of spaces, from: "Algebraic and geometric topology, Proc. Conf. New Brunswick/USA 1983", Lecture Notes in Math. 1126 Springer-Verlag (1985) 318-419

[50] F Waldhausen, Algebraic K-theory of spaces, concordance, and stable homotopy theory, from: "Algebraic topology and algebraic $K$-theory, Proc. Conf. Princeton, NJ (USA)", Ann. Math. Stud. 113 (1987) 392-417

[51] F Waldhausen et al, The stable parametrized $h$-cobordism theorem, in preparation 